TRANSACTIONS OF THE

AMERICAN MATHEMATICAL SOCIETY

Volume 362, Number 9, September 2010, Pages 4921-4954

S 0002-9947(10)05087-7

Article electronically published on April 28, 2010

\title{
THE EMERGENCE OF THE ELECTROSTATIC FIELD AS A FEYNMAN SUM IN RANDOM TILINGS WITH HOLES
}

\author{
MIHAI CIUCU
}

\begin{abstract}
We consider random lozenge tilings on the triangular lattice with holes $Q_{1}, \ldots, Q_{n}$ in some fixed position. For each unit triangle not in a hole, consider the average orientation of the lozenge covering it. We show that the scaling limit of this discrete field is the electrostatic field obtained when regarding each hole $Q_{i}$ as an electrical charge of magnitude equal to the difference between the number of unit triangles of the two different orientations inside $Q_{i}$. This is then restated in terms of random surfaces, yielding the result that the average over surfaces with prescribed height at the union of the boundaries of the holes is, in the scaling limit, a sum of helicoids.
\end{abstract}

\section{INTRODUCTION}

The study of correlations of holes in random tilings was launched by Fisher and Stephenson [10, who considered in particular the monomer-monomer correlation and obtained exact data suggesting rotational invariance in the scaling limit. Motivated by this, we studied correlations of a finite number of holes of various sizes and shapes in [1], 2] and [3], and found that in the scaling limit they are given by a multiplicative version of the superposition principle for energy in electrostatics. In this paper we consider the discrete field of average tile orientations in a random tiling with holes, and provide the proof of the fact that the electric field comes about as the scaling limit of this discrete field. This result was announced in [4].

Consider the unit equilateral triangular lattice drawn in the plane so that some of the lattice lines are vertical. The union of any two unit triangles that share an edge is called a lozenge. Inspired by Feynman's description of the reflection of light in [9, Ch. 2], and given the connections between lozenge tilings with holes and electrostatics discussed in 2, we construct a discrete vector field as follows. Let $\Delta_{1}, \ldots, \Delta_{n}$ be holes in the lattice whose boundaries are lattice triangles of even side-lengths. As one ranges over the set of lozenge tilings of the plane with these holes (a portion of such a tiling is illustrated in Figure 5.4(a)), any given left-pointing unit triangle $e$ in the complement of the holes is covered by a lozenge having one of three possible orientations: pointing in the polar direction 0 , the polar direction $2 \pi / 3$, or the polar direction $4 \pi / 3$. Define $\mathbf{F}(e)$ to be the average of these orientations over all tilings (the precise definitions are given in the next section).

Received by the editors January 13, 2009 and, in revised form, April 15, 2009.

2000 Mathematics Subject Classification. Primary 82B23, 82D99; Secondary 05A16, 60F99.

This research was supported in part by NSF grants DMS 0500616 and 0801625.

(C)2010 American Mathematical Society

Reverts to public domain 28 years from publication 

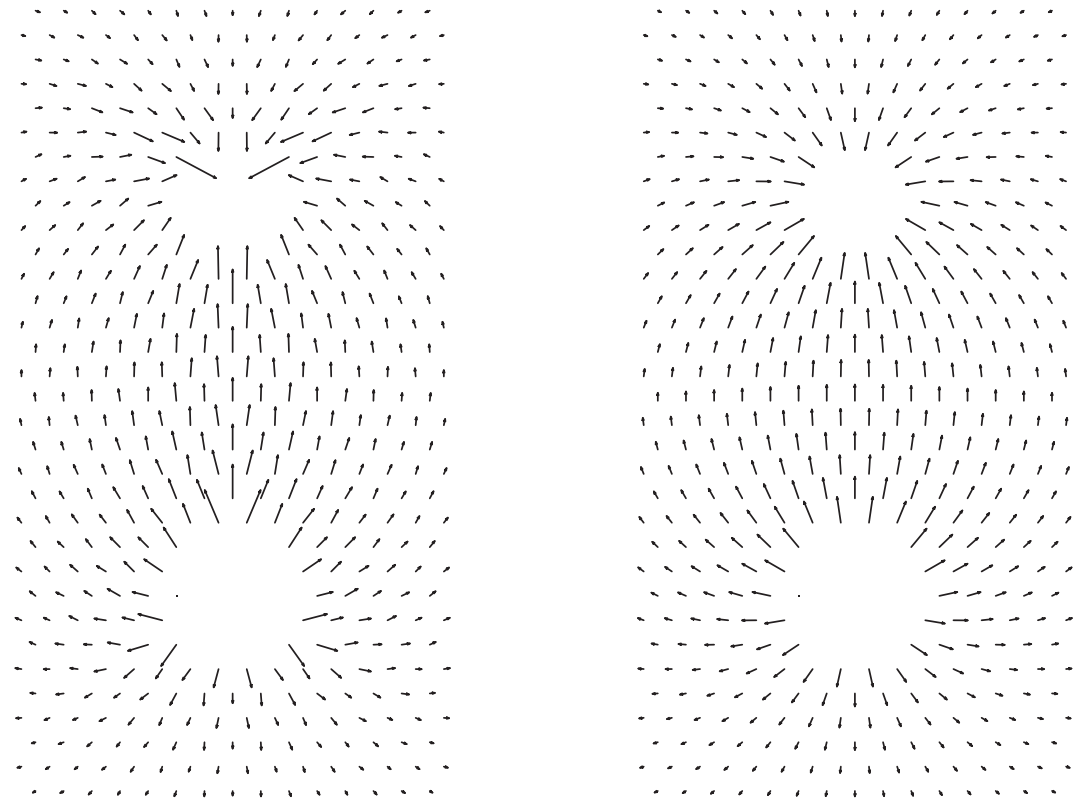

Now apply a homothety of modulus $1 / R$ to our lattice with holes, and "drag outward" the images of the holes and the image of $e$ so that they shrink to points $z_{1}, \ldots, z_{n}$ and, respectively, $z_{0}$ in the complex plane, as $R \rightarrow \infty$.

Our main result can be phrased as follows.

Theorem. As $R \rightarrow \infty$ we have

$$
\mathbf{F}(e) \sim \frac{3}{4 \pi R} \sum_{i=0}^{n} \operatorname{ch}\left(\Delta_{i}\right) \frac{\mathbf{r}_{i 0}}{\left|z_{0}-z_{i}\right|},
$$

where $\mathbf{r}_{i 0}$ is the unit vector pointing from $z_{i}$ in the direction of $z_{0}$ and $\operatorname{ch}(Q)$ denotes the difference between the number of right- and left-pointing unit triangles in the lattice region $Q$.

The figure above shows on the left an instance of the field $\mathbf{F}$ in the case of two oppositely oriented holes of side two, and on the right the corresponding Coulomb field that it approaches in the scaling limit.

In Section 5 (see Theorem 5.1) we phrase this result in terms of random surfaces. Lozenge tilings of the plane with holes lift to certain discrete multi-sheeted surfaces. The above result implies that the average over these surfaces (an instance of which is illustrated in Figure 5.5) converges in the scaling limit to a sum of helicoids (for lifting surfaces as in Figure 5.5, this limit surface is of the type depicted in Figure 5.7 (b); see 8 for an overview of a variety of settings in which helicoids arise). The key to proving this is that we obtain explicit formulas for the placement probabilities of lozenges in the scaling limit.

Phrased this way, our result is similar in spirit to the result of Cohn, Elkies and Propp [5] on the local statistics for random domino tilings of the Aztec diamond, where an explicit surface is found as the scaling limit of the normalized height function. Another related instance of an explicit limiting surface, concerning the placement probability of lozenges in a random tiling of a hexagon, was studied 
by Cohn, Larsen and Propp in [7]. In [6], Cohn, Kenyon and Propp show that the normalized average height function corresponding to domino tilings of regions that scale to an arbitrary simply connected region converges to the unique function that minimizes a certain surface tension integral. These authors also conjectured expressions for the local probability densities of domino configurations in large domino tilings. These conjectures were proved and generalized by Sheffield in [14]. Random surfaces corresponding to random dimer coverings of periodic bipartite planar lattices were studied extensively by Kenyon, Okounkov and Sheffield in [13.

One notable difference between our result and the above mentioned ones is the presence of holes. This causes the lifting surfaces to be multi-sheeted. The holes are also responsible for creating the field we are studying - without them the field would be zero.

It may also be worth noting that for instance in [7] the boundary is the boundary of a lattice hexagon, and remains of fixed size throughout the scaling process, while in our case the boundary consists of the union of finitely many boundaries of triangular holes, each of them shrinking to a point in the scaling limit. Related to this is the fact that, unlike the results from the literature mentioned above, we consider the average of un-normalized lifting surfaces - the average of the normalized ones is identically zero in the scaling limit.

\section{Definitions And Statement of Results}

Let $Q_{j}$ be a finite union of lattice triangular holes of side two with mutually disjoint interiors, for $j=1, \ldots, n$ (even when $Q_{j}$ is not connected, we still view it as a single generalized hole, often referred to as a multihole). The joint correlation $\hat{\omega}\left(Q_{1}, \ldots, Q_{n}\right)$ is defined in $[3$. (and recalled in Section 5 of the present paper) by means of limits of tori; the definition is readily extended to allow some of the $Q_{j}$ 's to be lozenges. It follows from that definition that if $L$ is any possible lozenge location, the probability that $L$ is occupied by a lozenge in a random lozenge tiling with holes at $Q_{1}, \ldots, Q_{n}$ is given by the ratio

$$
\frac{\hat{\omega}\left(L, Q_{1}, \ldots, Q_{n}\right)}{\hat{\omega}\left(Q_{1}, \ldots, Q_{n}\right)}
$$

(see Section 5 for the details). It thus follows that the vector $\mathbf{F}(e)=\mathbf{F}\left(e ; Q_{1}, \ldots, Q_{n}\right)$ described in the Introduction is given by

(1.1) $\mathbf{F}(e)=\frac{\hat{\omega}\left(L_{1}, Q_{1}, \ldots, Q_{n}\right)}{\hat{\omega}\left(Q_{1}, \ldots, Q_{n}\right)} \mathbf{e}_{1}+\frac{\hat{\omega}\left(L_{2}, Q_{1}, \ldots, Q_{n}\right)}{\hat{\omega}\left(Q_{1}, \ldots, Q_{n}\right)} \mathbf{e}_{2}+\frac{\hat{\omega}\left(L_{3}, Q_{1}, \ldots, Q_{n}\right)}{\hat{\omega}\left(Q_{1}, \ldots, Q_{n}\right)} \mathbf{e}_{3}$,

where $L_{1}, L_{2}$ and $L_{3}$ are the lozenge locations containing the left-pointing unit triangle $e$ and pointing in the $0,2 \pi / 3$ and $4 \pi / 3$ polar directions, respectively, and where the $\mathbf{e}_{j}$ 's are unit vectors pointing in the directions of the long diagonals of the $L_{j}$ 's.

Note that we can specify the location of any left- or right-pointing unit triangle (for short, we will call them left- and right-monomers) by indicating the location of the midpoint of its vertical side. The midpoints of the vertical sides of the unit triangles in our lattice can naturally be coordinatized by pairs of integers using a $60^{\circ}$ coordinate system with axes pointing in the polar directions $\pm \pi / 3$. 
Let $E_{a, b}$ be the east-pointing lattice triangle of side 2 whose central monomer has coordinates $(a, b)$. Let $W_{a, b}$ be the similarly defined west-pointing lattice triangle. We regard both of them as holes.

For any $q \in \mathbb{Q}$ and any strictly increasing list of integers $\mathbf{a}=\left(a_{1}, \ldots, a_{s}\right)$ for which $q a_{i} \in \mathbb{Z}$ and the $E_{a_{i}, q a_{i}}$ 's (equivalently, the $W_{a_{i}, q a_{i}}$ 's) are mutually disjoint, $i=1, \ldots, s$, define the multiholes $E_{\mathbf{a}}^{q}$ and $W_{\mathbf{a}}^{q}$ by

$$
\begin{aligned}
E_{\mathbf{a}}^{q} & =E_{a_{1}, q a_{1}} \cup \ldots \cup E_{a_{s}, q a_{s}}, \\
W_{\mathbf{a}}^{q} & =W_{a_{1}, q a_{1}} \cup \ldots \cup W_{a_{s}, q a_{s}} .
\end{aligned}
$$

For a hole $Q$ in the lattice, let $Q(x, y)$ stand for its translation by the vector $(x, y)$ in our coordinate system. We say that an integer divides a rational number if it divides the numerator of a lowest term representation of it.

We can now give the precise statement of our main result.

Theorem 1.1. Let $x_{0}^{(R)}, \ldots, x_{m}^{(R)}, y_{0}^{(R)}, \ldots, y_{m}^{(R)}, z_{0}^{(R)}, \ldots, z_{n}^{(R)}$ and $w_{0}^{(R)}, \ldots, w_{n}^{(R)}$ be sequences of integers so that $\lim _{R \rightarrow \infty} x_{i}^{(R)} / R=x_{i}, \lim _{R \rightarrow \infty} y_{i}^{(R)} / R=y_{i}$, $\lim _{R \rightarrow \infty} z_{j}^{(R)} / R=z_{j}$ and $\lim _{R \rightarrow \infty} w_{j}^{(R)} / R=w_{j}$ for $0 \leq i \leq m$ and $1 \leq j \leq n$. Assume the $\left(x_{i}, y_{i}\right)$ 's and $\left(z_{j}, w_{j}\right)$ 's are all distinct.

Then for any multiholes $E_{\mathbf{a}_{1}}^{q}, \ldots, E_{\mathbf{a}_{m}}^{q}$ and $W_{\mathbf{b}_{1}}^{q}, \ldots, W_{\mathbf{b}_{n}}^{q}$ with $3 \mid 1-q$, the field

$$
\mathbf{F}\left(x_{0}^{(R)}, y_{0}^{(R)}\right)=\mathbf{F}\left(x_{0}^{(R)}, y_{0}^{(R)} ; E_{\mathbf{a}_{1}}^{q}\left(x_{1}^{(R)}, y_{1}^{(R)}\right), \ldots, W_{\mathbf{b}_{n}}^{q}\left(z_{n}^{(R)}, w_{n}^{(R)}\right)\right)
$$

defined by (1.1) has orthogonal projections on our coordinate axes with asymptotics

$$
\begin{aligned}
& F_{x}\left(x_{0}^{(R)}, y_{0}^{(R)}\right)=\frac{3}{4 \pi R}\left\{\sum_{i=1}^{m} s_{i} \frac{2\left(x_{0}-x_{i}\right)+y_{0}-y_{i}}{\left(x_{0}-x_{i}\right)^{2}+\left(x_{0}-x_{i}\right)\left(y_{0}-y_{i}\right)+\left(y_{0}-y_{i}\right)^{2}}\right. \\
& \left.-\sum_{j=1}^{n} t_{j} \frac{2\left(x_{0}-z_{j}\right)+y_{0}-w_{j}}{\left(x_{0}-z_{j}\right)^{2}+\left(x_{0}-z_{j}\right)\left(y_{0}-w_{j}\right)+\left(y_{0}-w_{j}\right)^{2}}\right\}+o\left(\frac{1}{R}\right)
\end{aligned}
$$

and

$$
\begin{aligned}
& F_{y}\left(x_{0}^{(R)}, y_{0}^{(R)}\right)=\frac{3}{4 \pi R}\left\{\sum_{i=1}^{m} s_{i} \frac{x_{0}-x_{i}+2\left(y_{0}-y_{i}\right)}{\left(x_{0}-x_{i}\right)^{2}+\left(x_{0}-x_{i}\right)\left(y_{0}-y_{i}\right)+\left(y_{0}-y_{i}\right)^{2}}\right. \\
& \left.-\sum_{j=1}^{n} t_{j} \frac{x_{0}-z_{j}+2\left(y_{0}-w_{j}\right)}{\left(x_{0}-z_{j}\right)^{2}+\left(x_{0}-z_{j}\right)\left(y_{0}-w_{j}\right)+\left(y_{0}-w_{j}\right)^{2}}\right\}+o\left(\frac{1}{R}\right)
\end{aligned}
$$

where $s_{i}$ and $t_{j}$ are the lengths of $\mathbf{a}_{i}$ and $\mathbf{b}_{j}$, respectively.

Furthermore, for any $\epsilon>0$ and any bounded set $B$ in the plane, the implicit constants above are uniform over all choices of the limits for which each distance among the points $\left(x_{0}, y_{0}\right), \ldots,\left(z_{n}, w_{n}\right) \in B$ is at least $\epsilon$.

\footnotetext{
${ }^{1}$ The condition $3 \mid 1-q$ comes from the fact that in our proof of Theorem 1.1 we use 3 . Theorem 8.1], which has this condition as an assumption. As pointed out in [3], this is just a technical condition under which the calculations simplify. The special case when $q=1$ and the lists $\mathbf{a}_{i}$ and $\mathbf{b}_{j}$ consist of consecutive integers was the motivation for considering multiholes, as it is equivalent to having large triangular holes. Note that $3 \mid 1-q$ holds in this case.
} 
Note that in our oblique coordinate system the Euclidean distance between the points $(x, y)$ and $\left(x^{\prime}, y^{\prime}\right)$ is $\sqrt{\left(x-x^{\prime}\right)^{2}+\left(x-x^{\prime}\right)\left(y-y^{\prime}\right)+\left(y-y^{\prime}\right)^{2}}$, and the orthogonal projections of $(x, y)$ on the coordinate axes are $x+\frac{1}{2} y$ and $\frac{1}{2} x+y$. Note also that the multihole $E_{(0,2,4, \ldots, 2 s-2)}^{1}$ consists of a contiguous horizontal string of right-pointing triangular holes of side two. Due to forced lozenges in its complement, $E_{(0,2,4 \ldots, 2 s-2)}^{1}$ has precisely the same effect as the right-pointing triangular hole of side $2 s$ that contains it; a similar statement holds for $W$-multiholes. Since $\operatorname{ch}\left(E_{\mathbf{a}_{i}}^{q}\right)=2 s_{i}$ and $\operatorname{ch}\left(W_{\mathbf{b}_{j}}^{q}\right)=-2 t_{j}$, one sees that the theorem stated in the Introduction follows as a special case of Theorem 1.1.

\section{REDUCing the PROBlem to EXACT DETERMinant EVALUATIONS}

One crucial ingredient for proving the results of [3] was an exact determinant formula for the joint correlation of an arbitrary collection of disjoint lattice-triangular holes of size two. The arguments presented there prove in fact a more general statement, which we will need in the current paper.

Our determinant formula involves the coupling function $P(x, y), x, y \in \mathbb{Z}$, specified by

$$
P(x, y)=\frac{1}{2 \pi i} \int_{e^{2 \pi i / 3}}^{e^{4 \pi i / 3}} t^{-y-1}(-1-t)^{-x-1} d t, \quad x \leq-1
$$

and the symmetries $P(x, y)=P(y, x)=P(-x-y-1, x)$ (see Kenyon [12]), and the coefficients $U_{s}$ of its asymptotic series

$$
P(-3 r-1+a,-1+b) \sim \sum_{s=0}^{\infty}(3 r)^{-s-1} U_{s}(a, b), \quad r \rightarrow \infty, a, b \in \mathbb{Z} .
$$

Let $r(a, b)$ and $l(a, b)$ denote the right- and left-pointing monomers of coordinates $(a, b)$, respectively.

Proposition 2.1. Assume that $\left\{r\left(a_{1}, b_{1}\right), \ldots, r\left(a_{m}, b_{m}\right), l\left(c_{1}, d_{1}\right), \ldots, l\left(c_{n}, d_{n}\right)\right\}$ can be partitioned into subsets of size two so that the monomers in each subset share at least one vertex. Then if $m \geq n$ we have

$$
\hat{\omega}\left(r\left(a_{1}, b_{1}\right), \ldots, r\left(a_{m}, b_{m}\right), l\left(c_{1}, d_{1}\right), \ldots, l\left(c_{n}, d_{n}\right)\right)=\left|\operatorname{det}\left[\begin{array}{ll}
M_{P} & M_{U}
\end{array}\right]\right|,
$$

where

$$
M_{P}=\left[\begin{array}{ccc}
P\left(a_{1}-c_{1}, b_{1}-d_{1}\right) & \cdots & P\left(a_{1}-c_{n}, b_{1}-d_{n}\right) \\
P\left(a_{2}-c_{1}, b_{2}-d_{1}\right) & \cdots & P\left(a_{2}-c_{n}, b_{2}-d_{n}\right) \\
\vdots & & \vdots \\
P\left(a_{m}-c_{1}, b_{m}-d_{1}\right) & \cdots & P\left(a_{m}-c_{n}, b_{m}-d_{n}\right)
\end{array}\right]
$$

and $M_{U}=$

$$
\left[\begin{array}{ccccc}
U_{0}\left(a_{1}, b_{1}+1\right) & U_{0}\left(a_{1}+1, b_{1}\right) & \cdots & U_{\frac{m-n}{2}-1}\left(a_{1}, b_{1}+1\right) & U_{\frac{m-n}{2}-1}\left(a_{1}+1, b_{1}\right) \\
U_{0}\left(a_{2}, b_{2}+1\right) & U_{0}\left(a_{2}+1, b_{2}\right) & \cdots & U_{\frac{m-n}{2}-1}\left(a_{2}, b_{2}+1\right) & U_{\frac{m-n}{2}-1}\left(a_{2}+1, b_{2}\right) \\
\vdots & \vdots & & \vdots & \vdots \\
U_{0}\left(a_{m}, b_{m}+1\right) & U_{0}\left(a_{m}+1, b_{m}\right) & \cdots & U_{\frac{m-n}{2}-1}\left(a_{m}, b_{m}+1\right) & U_{\frac{m-n}{2}-1}\left(a_{m}+1, b_{m}\right)
\end{array}\right] .
$$

Proof. Since the set of monomers can be partitioned into pairs, $m+n$ is even; hence so is $m-n$. Thus we can prove the statement by induction on $m-n$ as follows. To relate a given configuration to one for which $m-n$ is 2 units smaller, bring in an 
extra hole, $W(3 r, 0)$, and let $r \rightarrow \infty$. The details go through just as in the proof of [3, Proposition 3.2].

In order to obtain the asymptotics of the field $\mathbf{F}(e)$ we need to understand the three coefficients in (1.1) when the holes are $E_{\mathbf{a}_{1}}^{(q)}, \ldots, E_{\mathbf{a}_{m}}^{(q)}, W_{\mathbf{b}_{1}}^{(q)}, \ldots, W_{\mathbf{b}_{n}}^{(q)}$. The asymptotics of the denominators in the special case $x_{1}^{(R)}=R x_{1}, \ldots, w_{n}^{(R)}=R w_{n}$, $x_{1}, \ldots, w_{n} \in 3 \mathbb{Z}$, is worked out in 3 , Theorem 8.1]. Proposition 2.1 supplies exact determinant expressions for the numerators. Each is the determinant of a block matrix whose blocks consist in turn of (mostly) $2 \times 2$ blocks. In order to list them it will be helpful to define the following five families of matrices.

Given two lists $\mathbf{a}=\left(a_{1}, \ldots, a_{s}\right)$ and $\mathbf{b}=\left(b_{1}, \ldots, b_{t}\right)$, let

$$
\begin{aligned}
& A_{x, y, z, w}(\mathbf{a}, \mathbf{b})= \\
& {\left[\begin{array}{cccc}
\ldots & P\left(x-z+a_{1}-b_{j}-1, y-w+q\left(a_{1}-b_{j}\right)-1\right) & P\left(x-z+a_{1}-b_{j}-2, y-w+q\left(a_{1}-b_{j}\right)\right) & \ldots \\
\cdots & P\left(x-z+a_{1}-b_{j}, y-w+q\left(a_{1}-b_{j}\right)-2\right) & P\left(x-z+a_{1}-b_{j}-1, y-w+q\left(a_{1}-b_{j}\right)-1\right) & \ldots \\
\ldots & P\left(x-z+a_{2}-b_{j}-1, y-w+q\left(a_{2}-b_{j}\right)-1\right) & P\left(x-z+a_{2}-b_{j}-2, y-w+q\left(a_{2}-b_{j}\right)\right) & \ldots \\
\ldots & P\left(x-z+a_{2}-b_{j}, y-w+q\left(a_{2}-b_{j}\right)-2\right) & P\left(x-z+a_{2}-b_{j}-1, y-w+q\left(a_{2}-b_{j}\right)-1\right) & \ldots \\
& \vdots & \vdots & \vdots \\
\vdots & & & \ldots \\
\ldots & P\left(x-z+a_{s}-b_{j}-1, y-w+q\left(a_{s}-b_{j}\right)-1\right) & P\left(x-z+a_{s}-b_{j}-2, y-w+q\left(a_{s}-b_{j}\right)\right) & \ldots \\
\cdots & P\left(x-z+a_{s}-b_{j}, y-w+q\left(a_{s}-b_{j}\right)-2\right) & P\left(x-z+a_{s}-b_{j}-1, y-w+q\left(a_{s}-b_{j}\right)-1\right) & \ldots
\end{array}\right]}
\end{aligned}
$$

(the display shows the $j$ th "column" of $2 \times 2$ blocks of $A_{x, y, z, w}(\mathbf{a}, \mathbf{b})$; there is one for each $j=1, \ldots, t)$.

The second family of matrices is

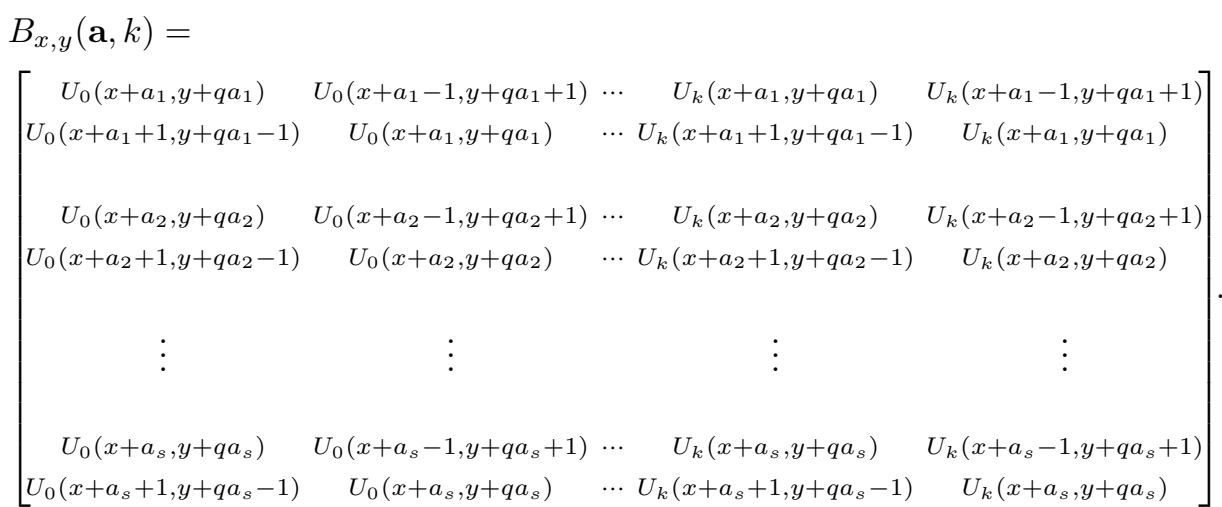

The remaining families consist of one-row or one-column matrices. The third is

$$
C_{x, y, z, w}(\mathbf{b})=\left[\begin{array}{llll}
\cdots & P\left(x-z-b_{j}, y-w-q b_{j}-1\right) & P\left(x-z-b_{j}-1, y-w-q b_{j}\right) & \ldots
\end{array}\right] .
$$


The fourth family is

$$
D_{x, y, z, w}(\mathbf{a})=\left[\begin{array}{c}
P\left(x-z+a_{1}-1, y-w+q a_{1}\right) \\
P\left(x-z+a_{1}, y-w+q a_{1}-1\right) \\
P\left(x-z+a_{2}-1, y-w+q a_{2}\right) \\
P\left(x-z+a_{2}, y-w+q a_{2}-1\right) \\
\vdots \\
P\left(x-z+a_{s}-1, y-w+q a_{s}\right) \\
P\left(x-z+a_{s}, y-w+q a_{s}-1\right)
\end{array}\right]
$$

and the fifth is

$$
G_{x, y}(k)=\left[U_{0}(x+1, y) U_{0}(x, y+1) \cdots U_{k}(x+1, y) U_{k}(x, y+1)\right] .
$$

Let $L_{1}(x, y), L_{2}(x, y)$, and $L_{3}(x, y)$ be the the lozenge locations pointing in the polar directions $0,2 \pi / 3,4 \pi / 3$, respectively, and containing the left-monomer $(x, y)$. Let $s_{i}$ and $t_{j}$ be the lengths of the lists $\mathbf{a}_{i}$ and $\mathbf{b}_{j}$, respectively, for $i=1, \ldots, m$, $j=1, \ldots, n$. Then Proposition 2.1 implie: 2

$$
\hat{\omega}\left(L_{1}\left(x_{0}^{(R)}, y_{0}^{(R)}\right), E_{\mathbf{a}_{1}}^{q}\left(x_{1}^{(R)}, y_{1}^{(R)}\right), \ldots, W_{\mathbf{b}_{n}}^{q}\left(z_{n}^{(R)}, w_{n}^{(R)}\right)\right)=\left|\operatorname{det} \bar{M}_{1}\right|,
$$

where

$$
\begin{aligned}
& {\left[\begin{array}{lll}
P(0,0) \quad C_{x_{0}^{(R)}, y_{0}^{(R)}, z_{1}^{(R)}, w_{1}^{(R)}}\left(\mathbf{b}_{1}\right) \quad \cdots
\end{array}\right.} \\
& \bar{M}_{1}=\left(\begin{array}{ccc}
D_{x_{1}^{(R)}, y_{1}^{(R)}, x_{0}^{(R)}, y_{0}^{(R)}}\left(\mathbf{a}_{1}\right) & A_{x_{1}^{(R)}, y_{1}^{(R)}, z_{1}^{(R)}, w_{1}^{(R)}}\left(\mathbf{a}_{1}, \mathbf{b}_{1}\right) & \cdots \\
\vdots & \vdots \\
& \\
D_{x_{m}^{(R)}, y_{m}^{(R)}, x_{0}^{(R)}, y_{0}^{(R)}}\left(\mathbf{a}_{m}\right) & A_{x_{m}^{(R)}, y_{m}^{(R)}, z_{1}^{(R)}, w_{1}^{(R)}}\left(\mathbf{a}_{m}, \mathbf{b}_{1}\right) & \cdots
\end{array}\right. \\
& \cdots \quad C_{x_{0}^{(R)}, y_{0}^{(R)}, z_{n}^{(R)}, w_{n}^{(R)}}\left(\mathbf{b}_{n}\right) \quad G_{x_{0}^{(R)}, y_{0}^{(R)}}(\nu) \\
& \cdots \quad A_{x_{1}^{(R)}, y_{1}^{(R)}, z_{n}^{(R)}, w_{n}^{(R)}}\left(\mathbf{a}_{1}, \mathbf{b}_{n}\right) \quad B_{x_{1}^{(R)}, y_{1}^{(R)}}\left(\mathbf{a}_{1}, \nu\right) \\
& \left.\cdots A_{x_{m}^{(R)}, y_{m}^{(R)}, z_{n}^{(R)}, w_{n}^{(R)}}\left(\mathbf{a}_{m}, \mathbf{b}_{n}\right) B_{x_{m}^{(R)}, y_{m}^{(R)}}\left(\mathbf{a}_{m}, \nu\right)\right]
\end{aligned}
$$

and $\nu=\sum_{i=1}^{m} s_{i}-\sum_{j=1}^{n} t_{j}-1$. We set $S=s_{1}+\cdots+s_{m}$ and $T=t_{1}+\cdots+t_{n}$.

${ }^{2}$ As in [3, Lemma 5.1], we use the fact that $E(x, y)$ can be replaced by the union of the two right-monomers $r(x-1, y)$ and $r(x, y-1)$, and $W(x, y)$ by $l(x+1, y) \cup l(x, y+1)$. 
Another application of Proposition 2.1 gives

$$
\hat{\omega}\left(E_{\mathbf{a}_{1}}^{q}\left(x_{1}^{(R)}, y_{1}^{(R)}\right), \ldots, W_{\mathbf{b}_{n}}^{q}\left(z_{n}^{(R)}, w_{n}^{(R)}\right)\right)=|\operatorname{det} M|,
$$

where 3

$$
M=\left(\bar{M}_{1}\right)_{[2 S+1] \backslash\{1\}}^{[2 S+1] \backslash\{1\}} .
$$

Denote

$$
p_{1}:=\frac{\hat{\omega}\left(L_{1}\left(x_{0}^{(R)}, y_{0}^{(R)}\right), E_{\mathbf{a}_{1}}^{q}\left(x_{1}^{(R)}, y_{1}^{(R)}\right), \ldots, W_{\mathbf{b}_{n}}^{q}\left(z_{n}^{(R)}, w_{n}^{(R)}\right)\right)}{\hat{\omega}\left(E_{\mathbf{a}_{1}}^{q}\left(x_{1}^{(R)}, y_{1}^{(R)}\right), \ldots, W_{\mathbf{b}_{n}}^{q}\left(z_{n}^{(R)}, w_{n}^{(R)}\right)\right)} .
$$

Throughout this paper $\zeta=e^{2 \pi i / 3}$.

Proposition 2.2. If $x_{0}^{(R)} / R \rightarrow x_{0}, \ldots, w_{n}^{(R)} / R \rightarrow w_{n}$ and $x_{0}^{(R)}=\alpha_{0}(\bmod 3), \ldots$, $w_{n}^{(R)}=\delta_{n}(\bmod 3)$, we have

$$
p_{1}=\frac{1}{3}+\frac{1}{2 \pi i R} \frac{\operatorname{det} M_{1}^{\prime \prime}}{\operatorname{det} M^{\prime \prime}}+o\left(\frac{1}{R}\right),
$$

where

$$
\begin{aligned}
& M_{1}^{\prime \prime}=\left[\begin{array}{ccc}
0 & \dot{C}_{x_{0}, y_{0}, z_{1}, w_{1}}^{\alpha_{0}, \beta_{0}, \gamma_{1}, \delta_{1}}\left(t_{1}\right) & \ldots \\
\dot{D}_{x_{1}, y_{1}, x_{0}, y_{0}}^{\alpha_{1}, \beta_{1}, \alpha_{0}, \beta_{0}}\left(s_{1}\right) & \dot{A}_{x_{1}, y_{1}, z_{1}, w_{1}}^{\alpha_{1}, \beta_{1}, w_{1}, \delta_{1}}\left(s_{1}, t_{1}\right) & \ldots \\
\vdots & & \\
& \vdots & \\
\dot{D}_{x_{m}, y_{m}, x_{0}, y_{0}}^{\alpha_{m}, \beta_{m}, \alpha_{0}, \beta_{0}}\left(s_{m}\right) & \dot{A}_{x_{m}, y_{m}, z_{1}, w_{1}}^{\alpha_{m}, \beta_{m}, \gamma_{m}, \delta_{1}}\left(s_{m}, t_{1}\right) & \ldots
\end{array}\right. \\
& \cdots \quad \dot{C}_{x_{0}, y_{0}, z_{n}, w_{n}}^{\alpha_{0}, \beta_{0}, \gamma_{n}, \delta_{n}}\left(t_{n}\right) \quad \dot{G}_{x_{0}, y_{0}}^{\alpha_{0}, \beta_{0}}(S-T-1) \\
& \cdots \quad \dot{A}_{x_{1}, y_{1}, z_{n}, w_{n}}^{\alpha_{1}, \beta_{1}, \gamma_{n}, \delta_{n}}\left(s_{1}, t_{n}\right) \quad \dot{B}_{x_{1}, y_{1}}^{\alpha_{1}, \beta_{1}}\left(s_{1}, S-T-1\right) \\
& \left.\cdots \quad \dot{A}_{x_{m}, y_{m}, z_{n}, w_{n}}^{\alpha_{m}, \beta_{m}, \gamma_{n}, \delta_{n}}\left(s_{m}, t_{n}\right) \quad \dot{B}_{x_{m}, y_{m}}^{\alpha_{m}, \beta_{m}}\left(s_{m}, S-T-1\right)\right]
\end{aligned}
$$

and

$$
\left.M^{\prime \prime}=\left(M_{1}^{\prime \prime}\right)[2 S+1] \backslash 1\right] \backslash\{1\},
$$

${ }^{3}$ We write $A_{I}^{J}$ for the submatrix of $A$ at the intersection of the rows with indices in $I$ with the columns with indices in $J ;[n]$ stands for $\{1, \ldots, n\}$. 
wit $\longdiv { 4 } \dot { A } _ { x , y , z , w } ^ { \alpha , \beta , \gamma , \delta } ( s , t ) =$

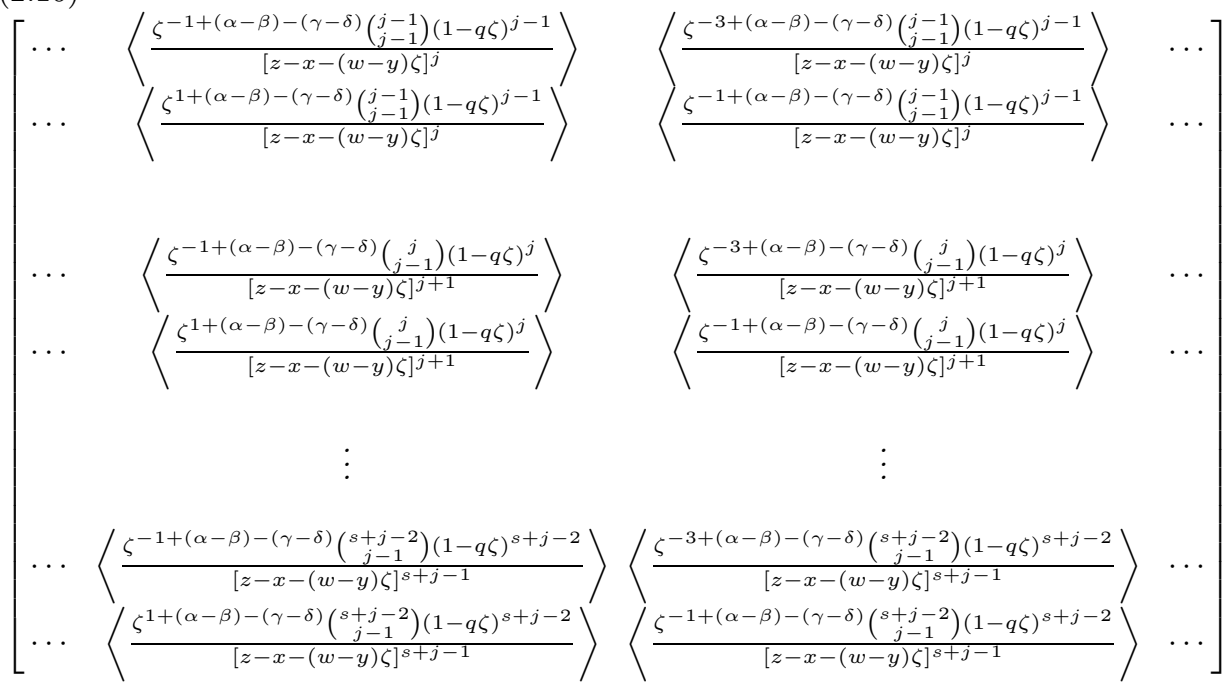

(there are $t$ pairs of such columns, one for each $j=1, \ldots, t$ ),

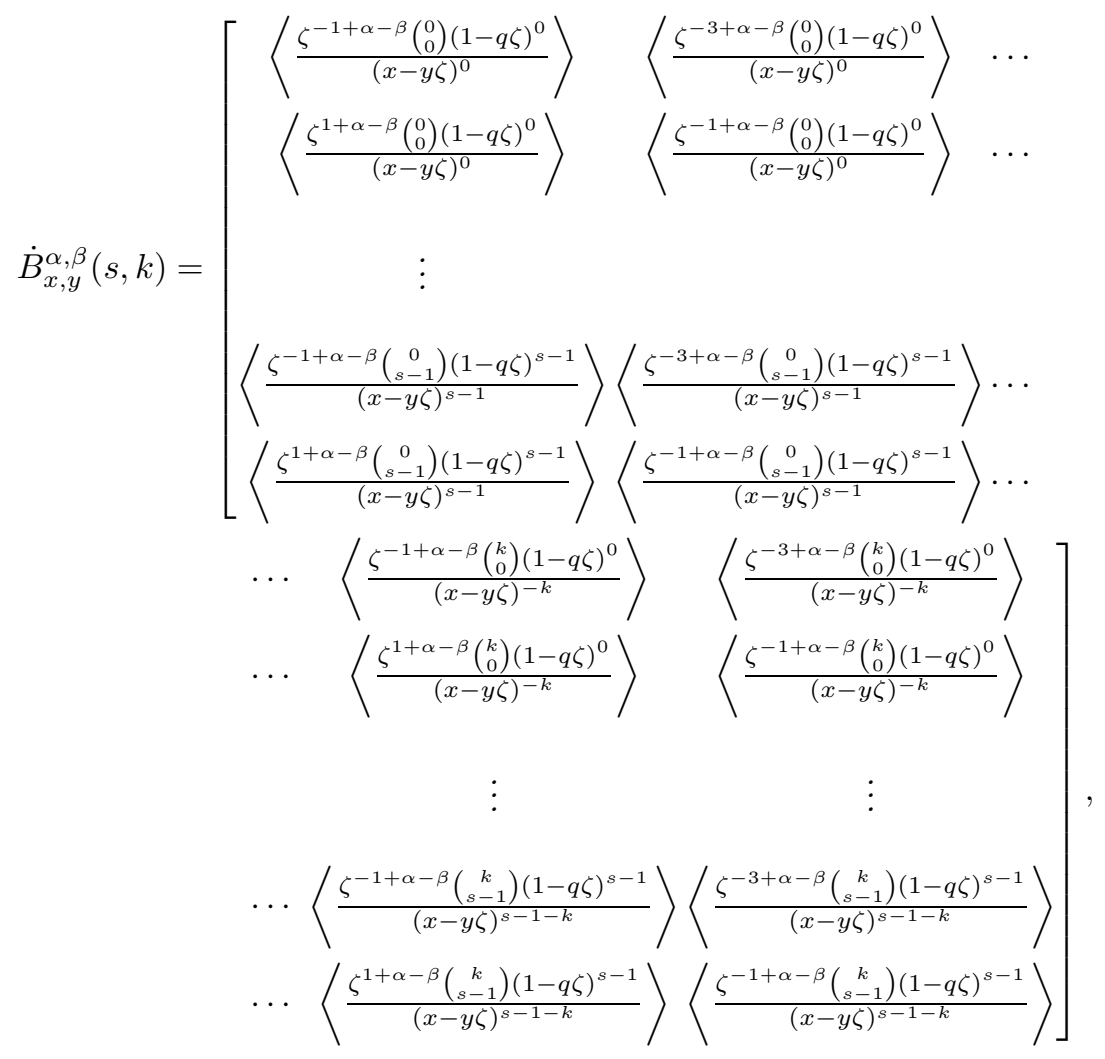

${ }^{4}$ Here and throughout the rest of the paper $\langle f(\zeta)\rangle$ stands for $f(\zeta)-f\left(\zeta^{-1}\right)$. 


$$
\begin{aligned}
& \left.\dot{C}_{x, y, z, w}^{\alpha, \beta, \gamma, \delta}(t)=\left[\cdots \quad\left\langle\frac{\zeta^{0+(\alpha-\beta)-(\gamma-\delta)}(1-q \zeta)^{j-1}}{[z-x-(w-y) \zeta]^{j}}\right\rangle\left\langle\frac{\zeta^{-2+(\alpha-\beta)-(\gamma-\delta)}(1-q \zeta)^{j-1}}{[z-x-(w-y) \zeta]^{j}}\right\rangle\right] \cdots\right] \\
& \dot{D}_{x, y, z, w}^{\alpha, \beta, \gamma, \delta}(s)=\left[\begin{array}{c}
\left\langle\frac{\zeta^{-2+(\alpha-\beta)-(\gamma-\delta)}(1-q \zeta)^{0}}{[z-x-(w-y) \zeta]}\right\rangle \\
\left\langle\frac{\zeta^{0+(\alpha-\beta)-(\gamma-\delta)}(1-q \zeta)^{0}}{[z-x-(w-y) \zeta]}\right\rangle \\
\left\langle\frac{\zeta^{-2+(\alpha-\beta)-(\gamma-\delta)}(1-q \zeta)^{1}}{[z-x-(w-y) \zeta]^{2}}\right\rangle \\
\left\langle\frac{\zeta^{0+(\alpha-\beta)-(\gamma-\delta)}(1-q \zeta)^{1}}{[z-x-(w-y) \zeta]^{2}}\right\rangle \\
\vdots \\
\left\langle\frac{\zeta^{-2+(\alpha-\beta)-(\gamma-\delta)}(1-q \zeta)^{s-1}}{[z-x-(w-y) \zeta]^{s}}\right\rangle \\
\left\langle\frac{\zeta^{0+(\alpha-\beta)-(\gamma-\delta)}(1-q \zeta)^{s-1}}{[z-x-(w-y) \zeta]^{s}}\right\rangle
\end{array}\right],
\end{aligned}
$$

and

$$
\dot{G}_{x, y}^{\alpha, \beta}(k)=\left[\left\langle\frac{\zeta^{0+\alpha-\beta}}{(x-y \zeta)^{0}}\right\rangle\left\langle\frac{\zeta^{-2+\alpha-\beta}}{(x-y \zeta)^{0}}\right\rangle\left\langle\frac{\zeta^{0+\alpha-\beta}}{(x-y \zeta)^{-1}}\right\rangle\left\langle\frac{\zeta^{-2+\alpha-\beta}}{(x-y \zeta)^{-1}}\right\rangle \cdots\left\langle\frac{\zeta^{0+\alpha-\beta}}{(x-y \zeta)^{-k}}\right\rangle\left\langle\frac{\zeta^{-2+\alpha-\beta}}{(x-y \zeta)^{-k}}\right\rangle\right] .
$$

Furthermore, for any $\epsilon>0$ and any bounded set B, the implicit constant in (2.13) is uniform for all choices of $x_{0}, \ldots, w_{n}$ for which $\left(x_{0}, y_{0}\right), \ldots,\left(z_{n}, w_{n}\right) \in B$ have all mutual distances at least $\epsilon$.

Note that (2.13)-(2.20) (plus 2 analogs) imply already, before evaluating the determinants, that in the scaling limit $\mathbf{F}(e)$ does not depend on the spacings between the side-two constituent holes of the multiholes $E_{\mathbf{a}_{i}}^{q}$ and $W_{\mathbf{b}_{j}}^{q}$ (although interestingly the independence of $q$ is not yet apparent).

Proof. By (2.12), (2.8) and (2.10) we have

$$
p_{1}=\left|\frac{\operatorname{det} \bar{M}_{1}}{\operatorname{det} M}\right|
$$

It readily follows from the integral expression $(2.1)$ for $P$ that $P(0,0)=1 / 3$. Regarding the first column of $\bar{M}_{1}$ as the sum of two vectors, one of which is $(1 / 3,0, \ldots, 0)^{T}$, and by using the linearity of the determinant yields

$$
p_{1}=\left|\frac{1}{3}+\frac{\operatorname{det} M_{1}}{\operatorname{det} M}\right|
$$

where

$$
M_{1}=\left.\bar{M}_{1}\right|_{(1,1) \text {-entry set to } 0} \text {. }
$$


In the special case $x_{1}^{(R)}=R x_{1}, \ldots, w_{n}^{(R)}=R w_{n}$ and $x_{1}=\cdots=w_{n}=0(\bmod 3)$, Lemma 5.3 of [3] shows how to write the main term in the asymptotics of $\operatorname{det} M$ as a determinant independent of $R$. This can be accomplished in the current case of general $x_{1}^{(R)}, \ldots, w_{n}^{(R)}$ by essentially the same arguments.

Namely, consider the following operation on a square matrix $X$ in which rows $i_{1}, \ldots, i_{k}$ are of the form $f\left(c_{1}\right), \ldots, f\left(c_{k}\right)$, respectively, for some vector function $f$ : Transform rows $i_{1}, \ldots, i_{k}$ of $X$ as

$$
\left[\begin{array}{c}
f\left(c_{1}\right) \\
f\left(c_{2}\right) \\
\cdot \\
\cdot \\
\cdot \\
f\left(c_{k}\right)
\end{array}\right] \mapsto\left[\begin{array}{c}
\mathcal{D}^{0} f\left(c_{1}\right) \\
\left(c_{2}-c_{1}\right) \mathcal{D}^{1} f\left(c_{1}\right) \\
\cdot \\
\cdot \\
\cdot \\
\left(c_{k}-c_{1}\right)\left(c_{k}-c_{2}\right) \ldots\left(c_{k}-c_{k-1}\right) \mathcal{D}^{k-1} f\left(c_{1}\right)
\end{array}\right],
$$

where $\mathcal{D}$ is Newton's divided difference operator, whose powers are defined inductively by $\mathcal{D}^{0} f=f$ and $\mathcal{D}^{r} f\left(c_{j}\right)=\left(\mathcal{D}^{r-1} f\left(c_{j+1}\right)-\mathcal{D}^{r-1} f\left(c_{j}\right)\right) /\left(c_{j+r}-c_{j}\right), r \geq 1$.

This operation has an obvious analog for columns.

As noted in [3, §5] (and as can be seen by looking at (2.11), (2.9), (2.3) and (2.4)), operation (2.24) can be applied a total of $2 m+2 n$ different times to the matrix $M$ : each row of block matrices in the expression for $M$ given by (2.11) and (2.9) provides two opportunities (along the odd-indexed rows and along the evenindexed ones), and each column consisting of $A$-blocks provides two more. Let $M^{\prime}$ be the matrix obtained from $M$ after applying these $2 m+2 n$ operations.

Since operations (2.24) preserve the determinant (see [3, Lemma 5.2]),

$$
\operatorname{det} M=\operatorname{det} M^{\prime} \text {. }
$$

Moreover, by construction, the $2 \times 2$ blocks of $M^{\prime}$ are obtained by replacing $R x_{1} \leftarrow$ $x_{1}^{(R)}, \ldots, R w_{n} \leftarrow w_{n}^{(R)}$ in formulas [3, (5.6)-(5.9)]. Theorem 4.1 and Proposition 4.5 and the arguments in the proof of [3, Proposition 5.3] then imply that 5

$$
\begin{aligned}
\operatorname{det}\left\lfloor\left\lfloor M^{\prime}\right\rfloor\right\rfloor=\left(\frac{1}{2 \pi i}\right)^{2 S} & \prod_{j=1}^{m} \prod_{1 \leq k<l \leq s_{j}}\left(a_{j k}-a_{j l}\right)^{2} \prod_{j=1}^{n} \prod_{\substack{1 \leq k<l \leq t_{j} \\
(2.26)}}\left(b_{j k}-b_{j l}\right)^{2} \\
& \times \operatorname{det}\left(M^{\prime \prime}\right) R^{2\left\{\sum_{1 \leq k<l \leq m} s_{k} s_{l}+\sum_{1 \leq k<l \leq n} t_{k} t_{l}-\sum_{k=1}^{m} \sum_{l=1}^{n} s_{k} t_{l}\right\}},
\end{aligned}
$$

where $\mathbf{a}_{k}=\left(a_{k, 1}, \ldots, a_{k, s_{k}}\right)$ and $\mathbf{b}_{l}=\left(b_{l, 1}, \ldots, b_{l, t_{l}}\right)$ for all $k$ and $l$.

By $(2.25)$ and (2.26) we get

$$
\begin{aligned}
& \operatorname{det} M=\left(\frac{1}{2 \pi i}\right)^{2 S} \prod_{j=1}^{m} \prod_{\begin{array}{l}
1 \leq k<l \leq s_{j} \\
\end{array}}\left(a_{j k}-a_{j l}\right)^{2} \prod_{j=1}^{n} \prod_{1 \leq k<l \leq t_{j}}\left(b_{j k}-b_{j l}\right)^{2} \\
& \quad \times \operatorname{det}\left(M^{\prime \prime}\right) R^{2\left\{\sum_{1 \leq k<l \leq m} s_{k} s_{l}+\sum_{1 \leq k<l \leq n} t_{k} t_{l}-\sum_{k=1}^{m} \sum_{l=1}^{n} s_{k} t_{l}\right\}} \\
& \quad+o\left(R^{2\left\{\sum_{1 \leq k<l \leq m} s_{k} s_{l}+\sum_{1 \leq k<l \leq n} t_{k} t_{l}-\sum_{k=1}^{m} \sum_{l=1}^{n} s_{k} t_{l}\right\}}\right) .
\end{aligned}
$$

\footnotetext{
${ }^{5}$ For a matrix $A$ whose entries depend on a large parameter, $\lfloor\lfloor A\rfloor\rfloor$ stands for the matrix obtained from it by replacing each entry by the dominant part in its asymptotics as the parameter approaches infinity.
} 
Theorem 4.1 and Proposition 4.5 imply that the implicit constant above is uniform in $x_{1}, \ldots, w_{n}$.

Next we turn to the asymptotics of $\operatorname{det} M_{1}$. Note that all the $2 m+2 n$ operations of type (2.24) we applied to $M$ are also well-defined as operations on $M_{1}$. Indeed, it is apparent from (2.9) and (2.3)-(2.6) that each of the $2 m+2 n$ times we applied (2.24), the extensions to $M_{1}$ of the involved rows or columns of $M$ are also of the form required by this operation. Let $M_{1}^{\prime}$ be the matrix obtained from $M_{1}$ after applying these $2 m+2 n$ operations.

A calculation similar to the one that gave (2.26) yields

$$
\begin{aligned}
\operatorname{det}\left\lfloor\left\lfloor M_{1}^{\prime}\right\rfloor\right\rfloor= & \left(\frac{1}{2 \pi i}\right)^{2 S+1} \prod_{j=1}^{m} \prod_{1 \leq k<l \leq s_{j}}\left(a_{j k}-a_{j l}\right)^{2} \prod_{j=1}^{n} \prod_{1 \leq k<l \leq t_{j}}\left(b_{j k}-b_{j l}\right)^{2} \\
& \times \operatorname{det}\left(M_{1}^{\prime \prime}\right) R^{2\left\{\sum_{1 \leq k<l \leq m} s_{k} s_{l}+\sum_{1 \leq k<l \leq n} t_{k} t_{l}-\sum_{k=1}^{m} \sum_{l=1}^{n} s_{k} t_{l}\right\}-1 .} .
\end{aligned}
$$

By the preservation of the determinant we get

$$
\begin{aligned}
\operatorname{det} M_{1}= & \left(\frac{1}{2 \pi i}\right)^{2 S+1} \prod_{j=1}^{m} \prod_{1 \leq k<l \leq s_{j}}\left(a_{j k}-a_{j l}\right)^{2} \prod_{j=1}^{n} \prod_{1 \leq k<l \leq t_{j}}\left(b_{j k}-b_{j l}\right)^{2} \\
& \times \operatorname{det}\left(M_{1}^{\prime \prime}\right) R^{2\left\{\sum_{1 \leq k<l \leq m} s_{k} s_{l}+\sum_{1 \leq k<l \leq n} t_{k} t_{l}-\sum_{k=1}^{m} \sum_{l=1}^{n} s_{k} t_{l}\right\}-1} \\
& +o\left(R^{2\left\{\sum_{1 \leq k<l \leq m} s_{k} s_{l}+\sum_{1 \leq k<l \leq n} t_{k} t_{l}-\sum_{k=1}^{m} \sum_{l=1}^{n} s_{k} t_{l}\right\}-1}\right),
\end{aligned}
$$

and the implicit constant is again uniform in $x_{1}, \ldots, w_{n}$ by Theorem 4.1 and Proposition 4.5. The statement of the proposition follows now by (2.22), (2.27) and (2.29).

The same analysis also proves the following result.

Proposition 2.3. If $x_{0}^{(R)} / R \rightarrow x_{0}, \ldots, w_{n}^{(R)} / R \rightarrow w_{n}$ and $x_{0}^{(R)}=\alpha_{0}(\bmod 3), \ldots$, $w_{n}^{(R)}=\delta_{n}(\bmod 3)$, we have

$$
\begin{aligned}
p_{2} & :=\frac{\hat{\omega}\left(L_{2}\left(x_{0}^{(R)}, y_{0}^{(R)}\right), E_{\mathbf{a}_{1}}^{q}\left(x_{1}^{(R)}, y_{1}^{(R)}\right), \ldots, W_{\mathbf{b}_{n}}^{q}\left(z_{n}^{(R)}, w_{n}^{(R)}\right)\right)}{\hat{\omega}\left(E_{\mathbf{a}_{1}}^{q}\left(x_{1}^{(R)}, y_{1}^{(R)}\right), \ldots, W_{\mathbf{b}_{n}}^{q}\left(z_{n}^{(R)}, w_{n}^{(R)}\right)\right)} \\
& =\frac{1}{3}+\frac{1}{2 \pi i R} \frac{\operatorname{det} M_{2}^{\prime \prime}}{\operatorname{det} M^{\prime \prime}}+o\left(\frac{1}{R}\right),
\end{aligned}
$$


where

$$
\begin{aligned}
& M_{2}^{\prime \prime}=\left[\begin{array}{ccc}
0 & \ddot{C}_{x_{0}, y_{0}, z_{1}, w_{1}}^{\alpha_{0}, \beta_{0}, \gamma_{1}, \delta_{1}}\left(t_{1}\right) & \ldots \\
\dot{D}_{x_{1}, y_{1}, x_{0}, y_{0}}^{\alpha_{1}, \beta_{1}, \alpha_{0}, \beta_{0}}\left(s_{1}\right) & \dot{A}_{x_{1}, y_{1}, z_{1}, w_{1}}^{\alpha_{1}, \beta_{1}, \gamma_{1}, \delta_{1}}\left(s_{1}, t_{1}\right) & \ldots \\
\vdots & & \\
\vdots & & \\
\dot{D}_{x_{m}, y_{m}, x_{0}, y_{0}}^{\alpha_{m}, \beta_{m}, \alpha_{0}, \beta_{0}}\left(s_{m}\right) & \dot{A}_{x_{m}, y_{m}, z_{1}, w_{1}}^{\alpha_{m}, \beta_{m}, \gamma_{1}, \delta_{1}}\left(s_{m}, t_{1}\right) & \ldots
\end{array}\right. \\
& \begin{array}{lcc}
\ldots & \ddot{C}_{x_{0}, y_{0}, z_{n}, w_{n}}^{\alpha_{0}, \beta_{0}, \gamma_{n}, \delta_{n}}\left(t_{n}\right) & \ddot{G}_{x_{0}, y_{0}}^{\alpha_{0}, \beta_{0}}(S-T-1) \\
\ldots & \dot{A}_{x_{1}, y_{1}, z_{n}, w_{n}}^{\alpha_{1}, \beta_{1}, \gamma_{n}, \delta_{n}}\left(s_{1}, t_{n}\right) & \dot{B}_{x_{1}, y_{1}}^{\alpha_{1}, \beta_{1}}\left(s_{1}, S-T-1\right)
\end{array} \\
& \left.\ldots \quad \dot{A}_{x_{m}, y_{m}, z_{n}, w_{n}}^{\alpha_{m}, \beta_{m}, \gamma_{n}, \delta_{n}}\left(s_{m}, t_{n}\right) \quad \dot{B}_{x_{m}, y_{m}}^{\alpha_{m}, \beta_{m}}\left(s_{m}, S-T-1\right)\right]
\end{aligned}
$$

$$
\ddot{C}_{x, y, z, w}^{\alpha, \beta, \gamma}(t)=\left[\cdots\left\langle\frac{\zeta^{-1+(\alpha-\beta)-(\gamma-\delta)}(1-q \zeta)^{j-1}}{[z-x-(w-y) \zeta]^{j}}\right\rangle\left\langle\frac{\zeta^{-3+(\alpha-\beta)-(\gamma-\delta)}(1-q \zeta)^{j-1}}{[z-x-(w-y) \zeta]^{j}}\right\rangle \cdots\right],
$$

and

$\ddot{G}_{x, y}^{\alpha, \beta}(k)=\left[\left\langle\frac{\zeta^{-1+\alpha-\beta}}{(x-y \zeta)^{0}}\right\rangle\left\langle\frac{\zeta^{-3+\alpha-\beta}}{(x-y \zeta)^{0}}\right\rangle\left\langle\frac{\zeta^{-1+\alpha-\beta}}{(x-y \zeta)^{-1}}\right\rangle\left\langle\frac{\zeta^{-3+\alpha-\beta}}{(x-y \zeta)^{-1}}\right\rangle \cdots\left\langle\frac{\zeta^{-1+\alpha-\beta}}{(x-y \zeta)^{-k}}\right\rangle\left\langle\frac{\zeta^{-3+\alpha-\beta}}{(x-y \zeta)^{-k}}\right\rangle\right]$.

The implicit constant above has the same uniformity property as in Proposition 2.2.

\section{Evaluation of the Determinants And Proof of TheOrem 1.1}

The orthogonal projections of the vectors $\mathbf{e}_{1}, \mathbf{e}_{2}$ and $\mathbf{e}_{3}$ of (1.1) on the $x$-axis of our oblique coordinate system are $\sqrt{3} / 2,-\sqrt{3} / 2$ and 0 , respectively. Thus the orthogonal projection of $\mathbf{F}$ on the $x$-axis is

$$
F_{x}=\frac{\sqrt{3}}{2}\left(p_{1}-p_{2}\right)
$$


By (2.13) and (2.30), the part about $F_{x}$ of Theorem 1.1 will follow provided we show that $\left(\operatorname{det} M_{1}^{\prime \prime}-\operatorname{det} M_{2}^{\prime \prime}\right) / \operatorname{det} M^{\prime \prime}$ evaluates to the expression that together with (3.1), (2.13) and (2.30) implies (1.2).

Proposition 3.1. The determinants of the matrices $M_{1}^{\prime \prime}$ given by (2.14) and (2.16)(2.20), $M_{2}^{\prime \prime}$ given by (2.31)-(2.33), and $M^{\prime \prime}$ given by (2.15) satisfy

$$
\frac{\operatorname{det} M_{1}^{\prime \prime}-\operatorname{det} M_{2}^{\prime \prime}}{\operatorname{det} M^{\prime \prime}}=i \sqrt{3}\left\{\sum_{k=1}^{m} s_{k} \frac{2\left(x_{0}-x_{k}\right)+y_{0}-y_{k}}{\left(x_{0}-x_{k}\right)^{2}+\left(x_{0}-x_{k}\right)\left(y_{0}-y_{k}\right)+\left(y_{0}-y_{k}\right)^{2}}\right.
$$

$$
\left.-\sum_{l=1}^{n} t_{l} \frac{2\left(x_{0}-z_{l}\right)+y_{0}-w_{l}}{\left(x_{0}-z_{l}\right)^{2}+\left(x_{0}-z_{l}\right)\left(y_{0}-w_{l}\right)+\left(y_{0}-w_{l}\right)^{2}}\right\} \text {. }
$$

A quick glance at (2.14) and (2.31) shows that $M_{1}^{\prime \prime}$ and $M_{2}^{\prime \prime}$ differ only in their first rows. Furthermore, by (2.18), (2.20), (2.32) and (2.33), the corresponding first row entries differ just by a factor of $\zeta^{-1}$. This makes it very tempting to write the numerator on the left hand side of (3.2) as a single determinant and try to use the method of factor exhaustion (after all, this was the method that proved successful in [3, where an explicit product expression for a special case of $\operatorname{det} M^{\prime \prime}$ is given; see [3, Theorem 8.1]). However, we were not able to prove (3.2) this way, despite getting frustratingly close (the only missing part was proving divisibility by one last type of linear polynomial divisor).

The proof below derives (3.2) by a certain limiting process from a convenient specialization of [3, Theorem 8.1]. This has the advantage of being significantly shorter than a possible proof by factor exhaustion. Still, a solution of the latter kind would be interesting, as it should also prove a conjectured two-parameter generalization of (3.2) that works in particular with $\zeta$ being an indeterminate.

Proof. We first prove the statement under the simplifying assumption that $\alpha_{0}=$ $\cdots=\delta_{n}=0$. In this case Theorem 8.1 of 3 implies that for any indeterminates $x_{1}, \ldots, x_{m}, y_{1}, \ldots, y_{m}, z_{1}, \ldots, z_{n}$ and $w_{1}, \ldots, w_{n}$ we have

$\operatorname{det} M^{\prime \prime}=\operatorname{det}$

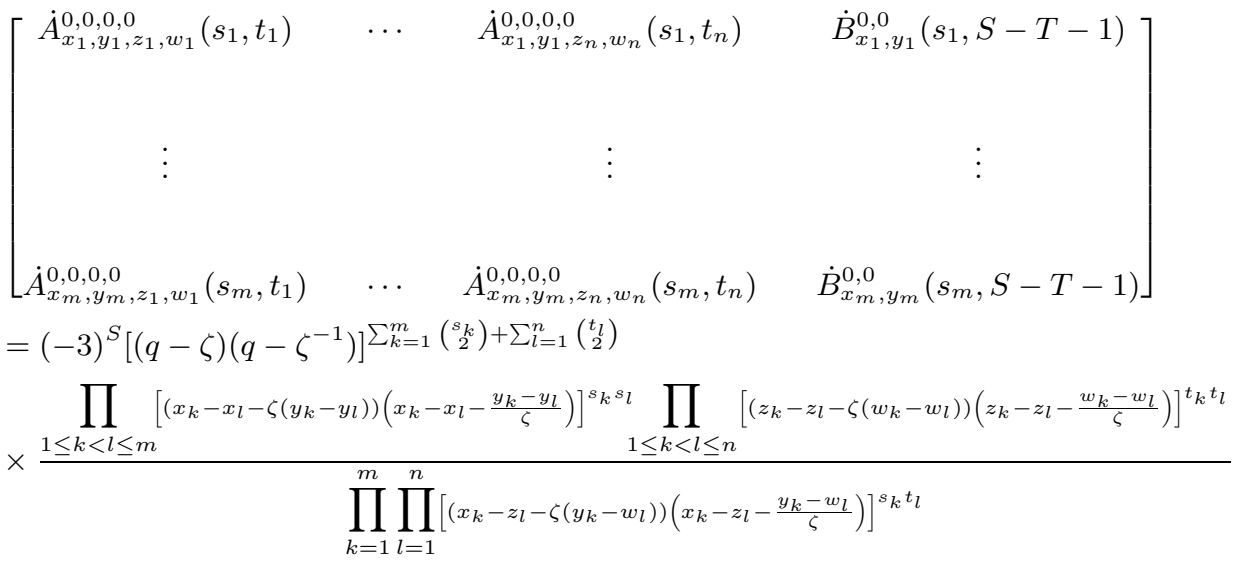

(the factor $(-3)^{S}$ comes from the factor $\left(\zeta^{2}-\zeta^{-2}\right)^{2 S}$ of [3, Theorem 8.1]). 
Let $\epsilon>0$. Note that the matrix in (3.3) depends on four lists of indeterminates, $x_{1}, \ldots, x_{m}, y_{1}, \ldots, y_{m}, z_{1}, \ldots, z_{n}$, and $w_{1}, \ldots, w_{n}$, and two lists of positive integers, $s_{1}, \ldots, s_{m}$ and $t_{1}, \ldots, t_{n}$. Replace the indeterminates as follows: the $x$-list by $x_{0}, x_{1}, \ldots, x_{m}$, the $y$-list by $y_{0}, y_{1}, \ldots, y_{m}$, the $z$-list by $x_{0}+\epsilon, z_{1}, \ldots, z_{n}$, and the $w$-list by $y_{0}, w_{1}, \ldots, w_{n}$. Also replace the $s$-list by $1, s_{1}, s_{2}, \ldots, s_{m}$, and the $t$-list by $1, t_{1}, t_{2}, \ldots, t_{n}$. Then (3.3) provides an explicit product expression for the determinant of the matrix

$$
\begin{aligned}
& M_{\epsilon}=\left[\begin{array}{cccc}
\left\langle\frac{\zeta^{-1}}{\epsilon}\right\rangle & \left\langle\frac{\zeta^{-3}}{\epsilon}\right\rangle & \ddot{C}_{x_{0}, y_{0}, z_{1}, w_{1}}^{0,0,0,0}\left(t_{1}\right) & \ldots \\
\left\langle\frac{\zeta}{\epsilon}\right\rangle & \left\langle\frac{\zeta^{-1}}{\epsilon}\right\rangle & \dddot{C}_{x_{0}, y_{0}, z_{1}, w_{1}}\left(t_{1}\right) & \ldots \\
\dddot{D}_{x_{1}, y_{1}, x_{0}+\epsilon, y_{0}}\left(s_{1}\right) & \ddot{D}_{x_{1}, y_{1}, x_{0}+\epsilon, y_{0}}\left(s_{1}\right) & \dot{A}_{x_{1}, y_{1}, z_{1}, w_{1}}^{0,0,0,0}\left(s_{1}, t_{1}\right) & \ldots \\
\vdots & \vdots & \vdots \\
\dddot{D}_{x_{m}, y_{m}, x_{0}+\epsilon, y_{0}}\left(s_{m}\right) & \ddot{D}_{x_{m}, y_{m}, x_{0}+\epsilon, y_{0}}\left(s_{m}\right) & \dot{A}_{x_{m}, y_{m}, z_{1}, w_{1}}^{0,0,0,0}\left(s_{m}, t_{1}\right) & \ldots
\end{array}\right. \\
& \ldots \quad \ddot{C}_{x_{0}, y_{0}, z_{n}, w_{n}}^{0,0,0,0}\left(t_{n}\right) \quad \ddot{G}_{x_{0}, y_{0}}^{0,0}(\nu) \\
& \ldots \quad \dddot{C}_{x_{0}, y_{0}, z_{n}, w_{n}}\left(t_{n}\right) \quad \dddot{G}_{x_{0}, y_{0}}(\nu) \\
& \cdots \quad \dot{A}_{x_{1}, y_{1}, z_{n}, w_{n}}^{0,0,0,0}\left(s_{1}, t_{n}\right) \quad \dot{B}_{x_{1}, y_{1}}^{0,0}\left(s_{1}, \nu\right),
\end{aligned}
$$

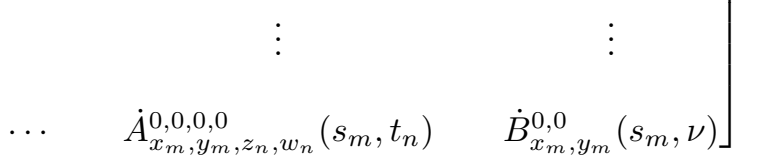

where $\nu=S-T-1$,

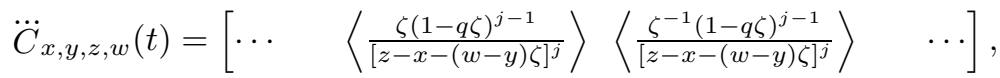

$$
\begin{aligned}
& \ddot{D}_{x, y, z, w}(s)=\left[\begin{array}{c}
\left\langle\frac{\zeta^{-3}(1-q \zeta)^{0}}{[z-x-(w-y) \zeta]}\right\rangle \\
\left\langle\frac{\zeta^{-1}(1-q \zeta)^{0}}{[z-x-(w-y) \zeta]}\right\rangle \\
\left\langle\frac{\zeta^{-3}(1-q \zeta)^{1}}{[z-x-(w-y) \zeta]^{2}}\right\rangle \\
\left\langle\frac{\zeta^{-1}(1-q \zeta)^{1}}{[z-x-(w-y) \zeta]^{2}}\right\rangle \\
\vdots \\
\left\langle\frac{\zeta^{-3}(1-q \zeta)^{s-1}}{[z-x-(w-y) \zeta]^{s}}\right\rangle \\
\left\langle\frac{\zeta^{-1}(1-q \zeta)^{s-1}}{[z-x-(w-y) \zeta]^{s}}\right\rangle
\end{array}\right],
\end{aligned}
$$




$$
\begin{gathered}
\text { MIHAI CIUCU } \\
\dddot{D}_{x, y, z, w}(s)=\left[\begin{array}{c}
\left\langle\frac{\zeta^{-1}(1-q \zeta)^{0}}{[z-x-(w-y) \zeta]}\right. \\
\left.\frac{\zeta(1-q \zeta)^{0}}{[z-x-(w-y) \zeta]}\right\rangle \\
\left\langle\begin{array}{c}
\frac{\zeta^{-1}(1-q \zeta)^{1}}{[z-x-(w-y) \zeta]^{2}} \\
\frac{\zeta(1-q \zeta)^{1}}{[z-x-(w-y) \zeta]^{2}}
\end{array}\right\rangle \\
\vdots \\
\left\langle\frac{\zeta^{-1}(1-q \zeta)^{s-1}}{[z-x-(w-y) \zeta]^{s}}\right\rangle \\
\left.\frac{\zeta(1-q \zeta)^{s-1}}{[z-x-(w-y) \zeta]^{s}}\right\rangle
\end{array}\right],
\end{gathered}
$$

and

$$
\begin{array}{r}
\dddot{G}_{x, y}(k)=\left[\left\langle\frac{\zeta}{(x-y \zeta)^{0}}\right\rangle\left\langle\frac{\zeta^{-1}}{(x-y \zeta)^{0}}\right\rangle\left\langle\frac{\zeta}{(x-y \zeta)^{-1}}\right\rangle\left\langle\frac{\zeta^{-1}}{(x-y \zeta)^{-1}}\right\rangle\right. \\
\left.\cdots\left\langle\frac{\zeta}{(x-y \zeta)^{-k}}\right\rangle\left\langle\frac{\zeta^{-1}}{(x-y \zeta)^{-k}}\right\rangle\right]
\end{array}
$$

The resulting product expression for $\operatorname{det} M_{\epsilon}$ has many common factors with the right hand side of (3.3), which simplify when taking $\operatorname{det} M_{\epsilon} / \operatorname{det} M^{\prime \prime}$. After simplification we get

$$
\begin{aligned}
& \frac{\operatorname{det} M_{\epsilon}}{\operatorname{det} M^{\prime \prime}}= \\
& -\frac{3}{\epsilon^{2}} \frac{\prod_{k=1}^{m}\left[\left(x_{0}-x_{k}-\zeta\left(y_{0}-y_{k}\right)\right)\left(x_{0}-x_{k}-\frac{y_{0}-y_{k}}{\zeta}\right)\right]^{s} \prod_{l=1}^{n}\left[\left(x_{0}+\epsilon-z_{l}-\zeta\left(y_{0}-w_{l}\right)\right)\left(x_{0}+\epsilon-z_{l}-\frac{y_{0}-w_{l}}{\zeta}\right)\right]^{t_{l}}}{\prod_{k=1}^{m}\left[\left(x_{k}-x_{0}-\epsilon-\zeta\left(y_{k}-y_{0}\right)\right)\left(x_{k}-x_{0}-\epsilon-\frac{y_{k}-y_{0}}{\zeta}\right)\right]^{s_{k}} \prod_{l=1}^{n}\left[\left(x_{0}-z_{l}-\zeta\left(y_{0}-w_{l}\right)\right)\left(x_{0}-z_{l}-\frac{y_{0}-w_{l}}{\zeta}\right)\right]^{t_{l}}} .
\end{aligned}
$$


Replace the first column in (3.4) by the negative of the sum of the first two columns. Using $\zeta^{3}=1,-\zeta^{-1}-\zeta^{-3}=\zeta^{-2}$, and $-\zeta-\zeta^{-1}=1$, we obtain

$$
\begin{aligned}
& -\operatorname{det} M_{\epsilon}=\mid \begin{array}{cccc}
\frac{\zeta-\zeta^{-1}}{\epsilon} & 0 & \ddot{C}_{x_{0}, y_{0}, z_{1}, w_{1}}^{0,0,0, t_{1}} & \ldots \\
0 & \frac{\zeta^{-1}-\zeta}{\epsilon} & \dddot{C}_{x_{0}, y_{0}, z_{1}, w_{1}}\left(t_{1}\right) & \ldots \\
\dot{D}_{x_{1}, y_{1}, x_{0}+\epsilon, y_{0}}^{0,0,0,0}\left(s_{1}\right) & \ddot{D}_{x_{1}, y_{1}, x_{0}+\epsilon, y_{0}}\left(s_{1}\right) & \dot{A}_{x_{1}, y_{1}, z_{1}, w_{1}}^{0,0,0,0}\left(s_{1}, t_{1}\right) & \ldots \\
\vdots & \vdots & \vdots \\
\dot{D}_{x_{m}, y_{m}, x_{0}+\epsilon, y_{0}}^{0,0,0,0}\left(s_{1}\right) & \ddot{D}_{x_{m}, y_{m}, x_{0}+\epsilon, y_{0}}\left(s_{1}\right) & \dot{A}_{x_{m}, y_{m}, z_{1}, w_{1}}^{0,0,0,0}\left(s_{m}, t_{1}\right) & \ldots
\end{array} \\
& \begin{array}{ccc}
\ldots & \ddot{C}_{x_{0}, 0, y_{0}, z_{n}, w_{n}}^{0,0,0}\left(t_{n}\right) & \ddot{G}_{x_{0}, y_{0}}^{0,0}(\nu) \\
\ldots & \dddot{C}_{x_{0}, y_{0}, z_{n}, w_{n}}\left(t_{n}\right) & \dddot{G}_{x_{0}, y_{0}}(\nu) \\
\ldots & \dot{A}_{x_{1}, y_{1}, z_{n}, w_{n}}^{0,0,0,0}\left(s_{1}, t_{n}\right) & \dot{B}_{x_{1}, y_{1}}^{0,0}\left(s_{1}, \nu\right) \\
& \vdots & \vdots \\
& &
\end{array}
\end{aligned}
$$

Denote the matrix on the right hand side above by $N=\left(N_{i j}\right)_{1<i, j<2 S+1}$. As $\epsilon \rightarrow 0$, the only diverging entries of $N$ are $N_{11}$ and $N_{22}$. It follows from (3.10) that

$$
\begin{gathered}
\operatorname{det} M_{\epsilon}=\frac{\left(\zeta-\zeta^{-1}\right)^{2}}{\epsilon^{2}} \operatorname{det} N_{[2 S+1] \backslash\{1,2\}}^{[2 S+1] \backslash\{1,2\}}+\frac{\zeta^{-1}-\zeta}{\epsilon}\left[\operatorname{det} \bar{N}_{[2 S+1] \backslash\{1\}}^{[2 S+1] \backslash\{1\}}-\operatorname{det} \bar{N}_{[2 S+1] \backslash\{2\}}^{[2 S+1] \backslash\{2\}}\right] \\
+O(1), \quad \epsilon \rightarrow 0,
\end{gathered}
$$

where $\bar{N}$ is the matrix obtained from $N$ by replacing $N_{11}$ and $N_{22}$ by 0 . But $\bar{N}_{[2 S+1] \backslash\{2\}}^{[2 S+1] \backslash\{2\}}$ is just the matrix $M_{2}^{\prime \prime}$ of $(2.31)$, and $N_{[2 S+1] \backslash\{1,2\}}^{[2 S+1]\{1,2\}}=M^{\prime \prime}$. Furthermore, by Lemma $3.2, \operatorname{det} \bar{N}_{[2 S+1] \backslash\{1\}}^{[2 S+1] \backslash\{1\}}=\operatorname{det} M_{1}^{\prime \prime}$. Therefore, (3.11) implies

$$
\frac{\operatorname{det} M_{\epsilon}}{\operatorname{det} M^{\prime \prime}}=-\frac{3}{\epsilon^{2}}+\frac{\zeta^{-1}-\zeta}{\epsilon} \frac{\operatorname{det} M_{1}^{\prime \prime}-\operatorname{det} M_{2}^{\prime \prime}}{\operatorname{det} M^{\prime \prime}}+O(1), \quad \epsilon \rightarrow 0 .
$$

Extracting the coefficient of $1 / \epsilon$ in the asymptotics of (3.9) as $\epsilon \rightarrow 0$ and comparing it with the second term in (3.12) gives (3.2).

Next we show how the case of general residues $\alpha_{0}, \ldots, \delta_{n}$ modulo 3 reduces to the above case of all zero residues.

Consider the general matrix $M_{1}^{\prime \prime}$ given by (2.14) and (2.16)-(2.20). Except for its first row and column, it consists of $2 \times 2$ blocks of the type in Lemma 3.3. Furthermore, the corresponding value of $a$ is constant over each block $\dot{A}_{x_{k}, y_{k}, z_{l}, w_{l}}^{\alpha_{k}, \beta_{k}, \gamma_{l}, \delta_{l}}\left(s_{k}, t_{l}\right)$ and equals $\left(\alpha_{k}-\beta_{k}\right)-\left(\gamma_{l}-\delta_{l}\right)$. It is also constant over each block $\dot{B}_{x_{k}, y_{k}}^{\alpha_{k}, y_{k}}\left(s_{k}, S-T-1\right)$ 
and equals $\alpha_{k}-\beta_{k}$. Thus in each Lemma 3.3 type $2 \times 2$ block in $M_{1}^{\prime \prime}$ the value of the $a$ of Lemma 3.3 is a difference of two quantities, the first being constant along each row of $M_{1}^{\prime \prime}$, and the second constant along each column of $M_{1}^{\prime \prime}$. Therefore Lemma 3.3 can be used to transform the general $M_{1}^{\prime \prime}$ matrix by row and column operations into the specialization of $M_{1}^{\prime \prime}$ when all residues are 0 except $\alpha_{0}$ and $\beta_{0}$, and the overall effect on the determinant is that it is multiplied by $\sigma$, where $\sigma \in\{1,-1\}$. The very same row and column operations transform $M_{2}^{\prime \prime}$ into $\left.M_{2}^{\prime \prime}\right|_{\alpha_{1}=0, \ldots, \delta_{n}=0}$ and $M^{\prime \prime}$ into $\left.M^{\prime \prime}\right|_{\alpha_{1}=0, \ldots, \delta_{n}=0}$, with the effect on their determinants being multiplication by the same $\sigma$. Therefore

$$
\frac{\operatorname{det} M_{1}^{\prime \prime}-\operatorname{det} M_{2}^{\prime \prime}}{\operatorname{det} M^{\prime \prime}}=\frac{\left.\operatorname{det} M_{1}^{\prime \prime}\right|_{\alpha_{1}=0, \ldots, \delta_{n}=0}-\left.\operatorname{det} M_{2}^{\prime \prime}\right|_{\alpha_{1}=0, \ldots, \delta_{n}=0}}{\left.\operatorname{det} M^{\prime \prime}\right|_{\alpha_{1}=0, \ldots, \delta_{n}=0}} .
$$

However, Lemma 3.4 provides determinant preserving operations that transform matrices $M_{1}^{\prime \prime}$ and $M_{2}^{\prime \prime}$ into their $\alpha_{0}=0, \beta_{0}=0$ specializations (note that $M^{\prime \prime}$ does not depend on $\alpha_{0}$ and $\beta_{0}$ ). Indeed, note that the only occurences of $\alpha_{0}$ and $\beta_{0}$ in $M_{1}^{\prime \prime}$ are in the first row and column, and there they only affect the exponent of $\zeta$ in the angular brackets that define the entries of the matrices of type $\dot{C}$ and $\dot{D}$. Furthermore, due to the fact that $\alpha_{0}$ and $\beta_{0}$ come first in the list of the upper four parameters of the $\dot{C}$-matrices, but last in the list of upper four parameters of the $\dot{D}$-matrices in (2.14), definitions (2.18) and (2.19) imply that the exponent of $\zeta$ in the angular brackets along the first row of $(2.14)$ is of the form $\left(\alpha_{0}-\beta_{0}\right)+\cdots$, while the corresponding exponent along the first column of (2.14) is of the form $-\left(\alpha_{0}-\beta_{0}\right)+\cdots$ (omitted terms are independent of $\alpha_{0}$ and $\left.\beta_{0}\right)$. Then by repeated use of Lemma 3.4 one can make the parts of these exponents that involve $\alpha_{0}$ and $\beta_{0}$ be 0 both along the first row and along the first column. A similar argument applies to the matrix $M_{2}^{\prime \prime}$. This completes the proof.

Lemma 3.2. $\operatorname{det} \bar{N}_{[2 S+1] \backslash\{1\}}^{[2 S+1] \backslash\{1\}}=\operatorname{det} M_{1}^{\prime \prime}$.

Proof. Repeated application of Lemma 3.4 transforms the first row and column of the matrix $\bar{N}_{[2 S+1] \backslash\{1\}}^{[2 S+1] \backslash\{1\}}$ (see (3.5), (3.7) and (3.8)) into the first row and column of the matrix $M_{1}^{\prime \prime}($ see $(2.18)-(2.20))$. All the other entries of $\bar{N}_{[2 S+1] \backslash\{1\}}^{[2 S+1] \backslash 1\}}$ and $\operatorname{det} M_{1}^{\prime \prime}$ agree and are left in agreement by the above applications of Lemma 3.4. This proves the claim.

Lemma 3.3. Let $f$ be a function defined at $\zeta$ and $\zeta^{-1}$, and let $a \in \mathbb{Z}$. Let

$$
A(a)=\left[\begin{array}{ll}
\left\langle\zeta^{a-1} f(\zeta)\right\rangle & \left\langle\zeta^{a-3} f(\zeta)\right\rangle \\
\left\langle\zeta^{a+1} f(\zeta)\right\rangle & \left\langle\zeta^{a-1} f(\zeta)\right\rangle
\end{array}\right] .
$$

Denote its rows by $R_{1}$ and $R_{2}$, and its columns by $C_{1}$ and $C_{2}$.

(a) Simultaneously replacing $\left\{R_{1} \leftarrow R_{2}, R_{2} \leftarrow-R_{1}-R_{2}\right\}$ turns matrix $A(a)$ into $A(a-1)$.

(b) Simultaneously replacing $\left\{C_{2} \leftarrow C_{1}, C_{1} \leftarrow-C_{1}-C_{2}\right\}$ turns matrix $A(a)$ into $A(a+1)$.

Proof. Since $\zeta^{3}=1$, the second row of $A(a)$ is the same as the first row of $A(a-1)$. The negative of the sum of the entries in the first column of $A(a)$ is

$$
\begin{gathered}
-\left(\zeta^{a-1} f(\zeta)-\zeta^{-a+1} f\left(\zeta^{-1}\right)\right)-\left(\zeta^{a+1} f(\zeta)-\zeta^{-a-1} f\left(\zeta^{-1}\right)\right) \\
=\zeta^{a} f(\zeta)-\zeta^{-a} f\left(\zeta^{-1}\right)=\left\langle\zeta^{a} f(\zeta)\right\rangle,
\end{gathered}
$$


as $-\zeta^{k}-\zeta^{k+2}=\zeta^{k+1}$ for all integers $k$. One similarly checks that the negative of the sum of the entries in the second column of $A(a)$ equals $\left\langle\zeta^{a-2} f(\zeta)\right\rangle$. This proves (a). Part (b) follows analogously.

By a similar calculation one can easily check the following.

Lemma 3.4. Let $f$ be a function defined at $\zeta$ and $\zeta^{-1}$, and let $\alpha, \beta, \gamma \in \mathbb{Z}$. Form the matrix

$$
\left[\begin{array}{ccc}
0 & \left\langle\zeta^{1+\alpha} f(\zeta)\right\rangle & \left\langle\zeta^{-1+\alpha} f(\zeta)\right\rangle \\
\left\langle\zeta^{-3+\beta} f(\zeta)\right\rangle & \left\langle\zeta^{-1+\gamma} f(\zeta)\right\rangle & \left\langle\zeta^{-3+\gamma} f(\zeta)\right\rangle \\
\left\langle\zeta^{-1+\beta} f(\zeta)\right\rangle & \left\langle\zeta^{1+\gamma} f(\zeta)\right\rangle & \left\langle\zeta^{-1+\gamma} f(\zeta)\right\rangle
\end{array}\right],
$$

denoting its rows by $R_{1}, R_{2}, R_{3}$ and its columns by $C_{1}, C_{2}, C_{3}$. Then the result of the simultaneous column operations $\left\{C_{2} \leftarrow-C_{2}-C_{3}, C_{3} \leftarrow C_{2}\right\}$, followed by the simultaneous row operations $\left\{R_{2} \leftarrow-R_{2}-R_{3}, R_{3} \leftarrow R_{2}\right\}$, is the matrix

$$
\left[\begin{array}{ccc}
0 & \left\langle\zeta^{\alpha} f(\zeta)\right\rangle & \left\langle\zeta^{-2+\alpha} f(\zeta)\right\rangle \\
\left\langle\zeta^{-2+\beta} f(\zeta)\right\rangle & \left\langle\zeta^{-1+\gamma} f(\zeta)\right\rangle & \left\langle\zeta^{-3+\gamma} f(\zeta)\right\rangle \\
\left\langle\zeta^{\beta} f(\zeta)\right\rangle & \left\langle\zeta^{1+\gamma} f(\zeta)\right\rangle & \left\langle\zeta^{-1+\gamma} f(\zeta)\right\rangle
\end{array}\right] .
$$

Proof of Theorem 1.1. Part (1.2) of the statement follows from (3.1), (2.13) and (3.2). By interchanging the roles of the coordinate axes we get (1.3).

Remark 3.5. Note that (2.27), (3.3), and the arguments in the paragraph preceding (3.13) extend the result of [3, Theorem 1.2] to the more general setting when the reference monomers in the multiholes have coordinates $\left(x_{i}^{(R)}, y_{i}^{(R)}\right)$ with $x_{i}^{(R)} / R \rightarrow$ $x_{i}, y_{i}^{(R)} / R \rightarrow y_{i}$, where the $x_{i}$ 's and $y_{i}$ 's are arbitrary real numbers ([3. Theorem 1.2] treats the special case $\left.x_{i}^{(R)}=R x_{i}, y_{i}^{(R)}=R y_{i}, x_{i}, y_{i} \in 3 \mathbb{Z}\right)$.

\section{A Finer integral ASymptotic}

Let $\mathcal{D}$ denote Newton's divided difference operator, whose powers are defined inductively by $\mathcal{D}^{0} f=f$ and $\mathcal{D}^{r} f\left(c_{j}\right)=\left(\mathcal{D}^{r-1} f\left(c_{j+1}\right)-\mathcal{D}^{r-1} f\left(c_{j}\right)\right) /\left(c_{j+r}-c_{j}\right)$, $r \geq 1$. We will need the following result on the asymptotics of the coupling function $P$ when acted on in the way indicated by powers of $\mathcal{D}$.

Theorem 4.1. Let $r_{n}$ and $s_{n}$ be integers so that $\lim _{n \rightarrow \infty} r_{n} / n=u, \lim _{n \rightarrow \infty} s_{n} / n=$ $v$, and $(u, v) \neq(0,0)$. Then for any integers $k, l \geq 0$ and any rational number $q$ with $3 \mid 1-q$ we have

$$
\begin{aligned}
\mathcal{D}_{y}^{l}\left\{\mathcal { D } _ { x } ^ { k } P \left(r_{n}+x\right.\right. & \left.\left.+y, s_{n}+q(x+y)\right)\left.\right|_{x=a_{1}}\right\}\left.\right|_{y=b_{1}} \\
= & \frac{1}{2 \pi i}\left(\begin{array}{c}
k+l \\
k
\end{array}\right)\left\langle\frac{\zeta^{r_{n}-s_{n}-1}(1-q \zeta)^{k+l}}{\left(-r_{n}+s_{n} \zeta\right)^{k+l+1}}\right\rangle+O\left(\frac{1}{n^{k+l+2}}\right),
\end{aligned}
$$

where $\zeta=e^{2 \pi i / 3}, \mathcal{D}_{x}^{k}$ acts with respect to a fixed integer sequence $a_{1}, a_{2}, \ldots$, and $\mathcal{D}_{y}^{l}$ acts with respect to an integer sequence $b_{1}, b_{2}, \ldots$ satisfying $q b_{j} \in \mathbb{Z}$ for all $j \geq 1$. Furthermore, for any open set $U$ containing the origin, the implicit constant above is uniform for all $(u, v) \notin U$.

We note that Proposition 7.1 of $\left[3\right.$ corresponds to the special case when $r_{n}=$ $-u n$ and $s_{n}=-v n$. It will be crucial in our proof of the scaling limit of the average lifting surface to know that the leading term on the right hand side of (4.1) is independent of the way $r_{n} / n$ and $s_{n} / n$ approach their limits. 
We deduce Theorem 4.1 from the following auxiliary results. Let $\mathcal{P}$ denote the counterclockwise oriented arc of the unit circle connecting $\zeta=e^{2 \pi i / 3}$ to -1 ; $\mathcal{P}[\zeta,-1)$ stands for this path minus the point -1 .

Lemma 4.2. Let $r_{n}$ and $s_{n}$ be integers, and assume $\lim _{n \rightarrow \infty} r_{n} / n=u \geq \epsilon>0$. Then if $q \in C^{0}(\mathcal{P}[\zeta,-1))$ has a pole of finite order at -1 , for any integer $k \geq 0$ we have

$$
\int_{\zeta}^{-1}(-1-t)^{r_{n}} t^{s_{n}}(t-\zeta)^{k} q(t) d t=O\left(\frac{1}{n^{k+1}}\right),
$$

where the implicit constant depends only on $k, \epsilon$ and $q(t)$ (in particular, it works for all $u \geq \epsilon$ and all integers $s_{n}$ ).

Proof. Let $I$ denote the integral above. Write $q(t)=\tilde{q}(t) /(t+1)^{l}$, with $l \geq 0$ and $\tilde{q} \in C^{0}(\mathcal{P})$. Making the change of variable $t=e^{i \theta}$ we obtain

$$
\begin{aligned}
I=(-1)^{r_{n}} \int_{\zeta}^{-1}(1+t)^{r_{n}-l} t^{s_{n}}(t-\zeta)^{k} \tilde{q}(t) d t \\
=(-1)^{r_{n}} \int_{2 \pi / 3}^{\pi}\left(2 \cos \frac{\theta}{2} e^{i \theta / 2}\right)^{r_{n}-l} e^{i \theta s_{n}}\left(e^{i \theta}-e^{2 \pi i / 3}\right)^{k} \tilde{q}\left(e^{i \theta}\right) i e^{i \theta} d \theta \\
=(-1)^{r_{n}} \int_{0}^{\pi / 3}\left[2 \cos \left(\frac{\pi}{3}+\frac{\tau}{2}\right) e^{i(\pi / 3+\tau / 2)}\right]^{r_{n}-l} e^{i(2 \pi / 3+\tau) s_{n}} \\
\times\left[e^{2 \pi i / 3}\left(e^{i \tau}-1\right)\right]^{k} \tilde{q}\left(e^{i(2 \pi / 3+\tau)}\right) i e^{i(2 \pi / 3+\tau)} d \tau .
\end{aligned}
$$

Since the graph of $2 \cos (\pi / 3+\tau / 2)$ is concave for $\tau \in[0, \pi / 3]$, it lies below its tangent at $\tau=0$. This implies

$$
2 \cos \left(\frac{\pi}{3}+\frac{\tau}{2}\right) \leq 1-\frac{\sqrt{3}}{2} \tau<1-\frac{\tau}{2}, \quad 0 \leq \tau \leq \pi / 3 .
$$

The elementary inequality $\left|e^{y}-1\right| \leq|y| e^{|y|}$ implies

$$
\left|e^{i \tau}-1\right| \leq \tau e^{\tau}<3 \tau, \quad \tau \in[0, \pi / 3] .
$$

The above two inequalities combined with (4.3) give

$$
\begin{aligned}
|I| & \leq \int_{0}^{\pi / 3}\left(1-\frac{\tau}{2}\right)^{r_{n}-l}(3 \tau)^{k}\left|\tilde{q}\left(e^{i(2 \pi / 3+\tau)}\right)\right| d \tau \\
& \leq 3^{k} \sup _{\tau \in \mathcal{P}}|\tilde{q}(t)| \int_{0}^{\pi / 3}\left(1-\frac{\tau}{2}\right)^{r_{n}-l} \tau^{k} d \tau .
\end{aligned}
$$

However, integration by parts implies

$$
\begin{aligned}
J(r, k) & :=\int_{0}^{\pi / 3}\left(1-\frac{\tau}{2}\right)^{r} \tau^{k} d \tau \\
& =-\frac{2}{r+1}\left(1-\frac{\pi}{6}\right)^{r+1}\left(\frac{\pi}{3}\right)^{k}+\frac{2 k}{r+1} J(r+1, k-1) .
\end{aligned}
$$

The repeated application of this, together with

$$
J(r+k, 0)=-\frac{2}{r+k+1}\left(1-\frac{\pi}{6}\right)^{r+k+1}+\frac{2}{r+k+2},
$$


shows that $J(r, k)$ is equal to a sum of $k+1$ terms each exponentially small in $r$, plus

$$
\frac{2}{r+k+2} \frac{(2 k)(2 k-2) \cdots 2}{(r+1)(r+2) \cdots(r+k)} .
$$

Since $r_{n}-l+1, \ldots, r_{n}-l+k+2 \geq \frac{1}{2} n u$ for $n$ large enough, it follows that the integral on the second line of (4.4) is majorized for $n$ large enough by $2^{2 k+3} k ! /(n u)^{k+1} \leq$ $2^{2 k+3} k ! /(\epsilon)^{k+1} 1 / n^{k+1}$, and the proof is complete.

Lemma 4.3. Let $q(t)=(t-\zeta)^{k} q_{1}(t)$, where $q_{1}(t) \in C^{1}(\mathcal{P}), k$ is a non-negative integer, and $q_{1}(\zeta) \neq 0$. Let $r_{n}, s_{n} \in \mathbb{Z}$ so that $\lim _{n \rightarrow \infty} r_{n} / n=u>0$.

(a) If $k=0$,

$$
\int_{\zeta}^{-1}(-1-t)^{r_{n}} t^{s_{n}} q(t) d t=\frac{\zeta^{s_{n}-r_{n}} q(\zeta)}{r_{n} \zeta-s_{n} \zeta^{-1}}+O\left(\frac{1}{n^{2}}\right)
$$

(b) If $k \geq 1$,

$$
\int_{\zeta}^{-1}(-1-t)^{r_{n}} t^{s_{n}} q(t) d t=\frac{1}{r_{n} \zeta-s_{n} \zeta^{-1}} \int_{\zeta}^{-1}(-1-t)^{r_{n}} t^{s_{n}} q^{\prime}(t) d t+O\left(\frac{1}{n^{k+2}}\right) .
$$

Furthermore, for any $\epsilon>0$, each implicit constant above can be chosen to be uniform for $u \in[\epsilon, \infty)$ and independent of $s_{n}$.

Proof. For $l=2,3, \ldots, 7$, define

$$
h_{l}(t):=\sum_{j \geq 0} \frac{1}{l+6 j}(t-\zeta)^{l+6 j}, \quad t \in \mathcal{P}[\zeta,-1) .
$$

One readily checks that the Taylor series expansions of $\ln t$ and $\ln (-1-t)$ around $t=\zeta$ can then be written as

$$
\ln t=\ln \zeta+\zeta^{-1}(t-\zeta)-\zeta^{-1} h_{4}+\zeta^{-1} h_{7}-\zeta h_{2}+\zeta h_{5}+h_{3}-h_{6}
$$

and

$$
\ln (-1-t)=\ln (-1-\zeta)-\zeta(t-\zeta)-\zeta h_{4}-\zeta h_{7}-\zeta^{-1} h_{2}-\zeta^{-1} h_{5}-h_{3}-h_{6} .
$$

Therefore we have

$$
\begin{aligned}
& r \ln (-1-t)+s \ln t=r \ln (-1-\zeta)+s \ln \zeta+\left(s \zeta^{-1}-r \zeta\right)(t-\zeta) \\
& \quad+\left(-r \zeta^{-1}-s \zeta\right) h_{2}(t)+(-r+s) h_{3}(t)+\left(-r \zeta-s \zeta^{-1}\right) h_{4}(t) \\
& \quad+\left(-r \zeta^{-1}+s \zeta\right) h_{5}(t)+(-r-s) h_{6}(t)+\left(-r \zeta+s \zeta^{-1}\right) h_{7}(t), \quad t \in \mathcal{P}[\zeta,-1) .
\end{aligned}
$$

Let $r, s \in \mathbb{Z}$ and denote

$$
I(r, s):=\int_{\zeta}^{-1}(-1-t)^{r} t^{s} q(t) d t
$$


Using (4.8) and $\zeta^{3}=1$, integration by parts gives

$$
\begin{aligned}
& I(r, s)=\int_{\zeta}^{-1} e^{r \ln (-1-t)+s \ln t} q(t) d t \\
& =\zeta^{s-r} \int_{\zeta}^{-1} e^{\left(s \zeta^{-1}-r \zeta\right)(t-\zeta)} e^{\left(-r \zeta^{-1}-s \zeta\right) h_{2}+\cdots+\left(-r \zeta+s \zeta^{-1}\right) h_{7}} q(t) d t \\
& =\zeta^{s-r}\left\{\left.\frac{e^{\left(s \zeta^{-1}-r \zeta\right)(t-\zeta)}}{s \zeta^{-1}-r \zeta} e^{b(t)} q(t)\right|_{\zeta} ^{-1}-\frac{1}{s \zeta^{-1}-r \zeta} \int_{\zeta}^{-1} e^{\left(s \zeta^{-1}-r \zeta\right)(t-\zeta)}\right. \\
& \left.\times\left[\left(\left(-r \zeta^{-1}-s \zeta\right) h_{2}^{\prime}(t)+\cdots+\left(-r \zeta+s \zeta^{-1}\right) h_{7}^{\prime}(t)\right) e^{b(t)} q(t)+e^{b(t)} q^{\prime}(t)\right] d t\right\},
\end{aligned}
$$

where

$$
\begin{aligned}
b(t) & :=\left(-r \zeta^{-1}-s \zeta\right) h_{2}(t)+(-r+s) h_{3}(t)+\left(-r \zeta-s \zeta^{-1}\right) h_{4}(t) \\
& +\left(-r \zeta^{-1}+s \zeta\right) h_{5}(t)+(-r-s) h_{6}(t)+\left(-r \zeta+s \zeta^{-1}\right) h_{7}(t) .
\end{aligned}
$$

Since $\zeta^{s-r} e^{\left(s \zeta^{-1}-r \zeta\right)(t-\zeta)} e^{b(t)}=(-1-t)^{r} t^{s}$, we see that the upper limit in the first term of the expression in the large curly braces above equals 0 whenever $r>k$. Thus, for $r>k$ we obtain

$$
\begin{gathered}
I(r, s)=\frac{\zeta^{s-r} q(\zeta)}{r \zeta-s \zeta^{-1}}+\zeta^{s-r}\left\{\frac{-r \zeta^{-1}-s \zeta}{r \zeta-s \zeta^{-1}} \int_{\zeta}^{-1} e^{\left(s \zeta^{-1}-r \zeta\right)(t-\zeta)} e^{b(t)} q(t) h_{2}^{\prime}(t) d t+\cdots\right. \\
\left.\quad+\frac{-r \zeta+s \zeta^{-1}}{r \zeta-s \zeta^{-1}} \int_{\zeta}^{-1} e^{\left(s \zeta^{-1}-r \zeta\right)(t-\zeta)} e^{b(t)} q(t) h_{7}^{\prime}(t) d t\right\} \\
\quad+\frac{\zeta^{s-r}}{r \zeta-s \zeta^{-1}} \int_{\zeta}^{-1} e^{\left(s \zeta^{-1}-r \zeta\right)(t-\zeta)} e^{b(t)} q^{\prime}(t) d t .
\end{gathered}
$$

If $|r| \geq|s|$ we have

$$
\left|\frac{-r \zeta^{-1}-s \zeta}{r \zeta-s \zeta^{-1}}\right|=\left|\frac{1+s / r \zeta^{-1}}{1-s / r \zeta}\right| \leq \frac{1+|s / r|}{|\operatorname{Re}(1-s / r \zeta)|} \leq \frac{2}{1 / 2}=4 .
$$

A similar argument shows that the above inequality holds in fact also when $|s| \geq|r|$. All six fractions in front of the integrals in the expression in curly braces above are thus seen to be majorized in absolute value by 4 .

Regard $I\left(r_{n}, s_{n}\right)$ as the sum of the three quantities provided by (4.9). To deduce part (a) of the lemma, assume $k=0$. All six terms of the second quantity are $O\left(1 / n^{2}\right)$ thanks to (4.10) and an application of Lemma 4.2 with $k=1$ (which applies since $h_{2}^{\prime}(t), \ldots, h_{7}^{\prime}(t)$ are all of the form $(t-\zeta) g(t)$, where $g \in C^{\infty}(\mathcal{P}[\zeta,-1))$ has a simple pole at $t=-1$ ). Finally, the third quantity provided by (4.9) is also $O\left(1 / n^{2}\right)$, due to the fraction in front of the integral and another application of Lemma 4.2 with $k=0$.

For part (b), assume $k \geq 1$. Then $q(\zeta)=0$, and (4.9) provides an expression for $I\left(r_{n}, s_{n}\right)$ as a sum of just two quantities. All six terms in the first quantity are $O\left(1 / n^{k+2}\right)$ due to (4.10) and Lemma 4.2 applied with $k$ replaced by $k+1$ (as explained in the previous paragraph, this unit increment comes about by the presence of the $h_{l}^{\prime}(t)$ factors in the integrands). This proves (4.6). 
The uniformity of the implicit constant follows because both the majorant in (4.10) and the implicit constant in (4.2) are uniform.

Repeated application of part (b) of the above lemma and one final application of part (a) yields the following result.

Proposition 4.4. Let $q(t)=(t-\zeta)^{k} q_{1}(t)$, where $k \geq 0, q_{1} \in C^{k+1}(\mathcal{P}[\zeta,-1))$ has a pole of finite order at $t=-1$, and $q_{1}(\zeta) \neq 0$. Let $r_{n}, s_{n} \in \mathbb{Z}$ so that $\lim _{n \rightarrow \infty} r_{n} / n=u>0$. Then

$$
\int_{\zeta}^{-1}(-1-t)^{r_{n}} t^{s_{n}} q(t) d t=\frac{\zeta^{s_{n}-r_{n}} q^{(k)}(\zeta)}{\left(r_{n} \zeta-s_{n} \zeta^{-1}\right)^{k+1}}+O\left(\frac{1}{n^{k+2}}\right) .
$$

Furthermore, for any $\epsilon>0$ the implicit constant is uniform for $u \in[\epsilon, \infty)$ and independent of $s_{n}$.

Proof of Theorem 4.1. Suppose first that $u<0$. Thus (2.1) applies for large enough $n$, and for $3 \mid 1-q[3,(7.11)]$ and [3] Lemma 7.4] give

$$
\begin{aligned}
& \left.\mathcal{D}_{y}^{l}\left\{\left.\mathcal{D}_{x}^{k} P\left(r_{n}+x+y, s_{n}+q(x+y)\right)\right|_{x=a_{1}}\right\}\right|_{y=b_{1}} \\
& =\frac{1}{2 \pi i}\left\langle\int _ { \zeta } ^ { - 1 } ( - 1 - t ) ^ { - r _ { n } } t ^ { - s _ { n } } \left\{\frac{1}{k ! l !}\left(\zeta-q \zeta^{-1}\right)^{k+l}(t-\zeta)^{k+l}\right.\right. \\
& \left.\left.\quad+c_{k+l+1}(t-\zeta)^{k+l+1}+\cdots\right\} d t\right\rangle .
\end{aligned}
$$

Proposition 4.4 applied to the right hand side above yields

$$
\left.\mathcal{D}_{y}^{l}\left\{\left.\mathcal{D}_{x}^{k} P\left(r_{n}+x+y, s_{n}+q(x+y)\right)\right|_{x=a_{1}}\right\}\right|_{y=b_{1}}
$$

$$
\frac{1}{2 \pi i}\left\langle\frac{\zeta^{s_{n}-r_{n}} \frac{1}{k ! l !}\left(\zeta-q \zeta^{-1}\right)^{k+l}(k+l) !}{\left(r_{n} \zeta-s_{n} \zeta^{-1}\right)^{k+l+1}}\right\rangle+O\left(\frac{1}{n^{k+l+2}}\right),
$$

which is just what (4.1) states. The uniformity of the implicit constant above follows by the uniformity of the implicit constant in (4.11).

Since $(u, v) \neq(0,0)$, at least one of $u<0, v<0$, and $-u-v<0$ is true. The symmetries $P(\alpha, \beta)=P(-\alpha-\beta-1, \alpha)$ and $P(\alpha, \beta)=P(\beta, \alpha)$ of the coupling function allow one to use the same arguments that proved the case $u<0$ to deduce the other two cases (see the proof of Proposition 7.1 in 3 for details).

Proposition 4.5. Let $r_{n}, s_{n} \in \mathbb{Z}$ so that $\lim _{n \rightarrow \infty} r_{n} / n=u$ and $\lim _{n \rightarrow \infty} s_{n} / n=v$. Then for any integers $k, l \geq 0$ and any rational number $q$ with $3 \mid 1-q$ we have

$$
\left.\mathcal{D}_{x}^{k} U_{l}\left(r_{n}+x, s_{n}+q x\right)\right|_{x=a_{1}}=\frac{1}{2 \pi i}\left(\begin{array}{l}
l \\
k
\end{array}\right)\left\langle\zeta^{r_{n}-s_{n}-1}(1-q \zeta)^{k}\left(r_{n}-s_{n} \zeta\right)^{l-k}\right\rangle
$$

$$
+O\left(n^{l-k-1}\right) \text {, }
$$

where $\mathcal{D}_{x}^{k}$ acts with respect to some fixed integer sequence $a_{1}, a_{2}, \ldots$ Given any bounded set $B$ in the plane, the implicit constant can be chosen so that it is uniform for $(u, v) \in B$. 
Proof. By [3, (6.8)] one has

$$
\begin{aligned}
U_{l}(a, b)= & \frac{1}{2 \pi i}\left\langle\zeta^{a-b-1}(a-b \zeta)^{l}\right\rangle \\
& + \text { monomials in } a \text { and } b \text { of joint degree }<l .
\end{aligned}
$$

This implies, for $3 \mid 1-q$, that

$$
\begin{aligned}
& U_{l}\left(r_{n}+x, s_{n}+q x\right)=\frac{1}{2 \pi i}\left\langle\zeta^{r_{n}-s_{n}-1}\left[(1-q \zeta) x+\left(r_{n}-s_{n} \zeta\right)\right]^{l}\right\rangle \\
& +\sum_{\substack{\alpha, \beta>0 \\
\alpha+\beta<l}} c_{\alpha, \beta}\left(r_{n}+x\right)^{\alpha}\left(s_{n}+q x\right)^{\beta},
\end{aligned}
$$

where $c_{\alpha, \beta}$ is independent of $r_{n}$ and $s_{n}$ for all $\alpha$ and $\beta$.

On the other hand, the argument that proved [3, Lemma 6.4] implies that for any constants $A, B, C \in \mathbb{C}$

$$
\left.\mathcal{D}_{x}^{k}\left(A x+B r_{n}+C s_{n}\right)^{l}\right|_{x=a_{1}}=\left(\begin{array}{l}
l \\
k
\end{array}\right) A^{k}\left(B r_{n}+C s_{n}\right)^{l-k}+O\left(n^{l-k-1}\right),
$$

with implicit constant uniform for $(u, v)$ ranging over any bounded set. Combining (4.14) and (4.15) yields the statement of the proposition.

\section{INTERPRETATION IN TERMS OF HEIGHT FUNCTIONS}

Lozenge tilings of regions with no holes are well known to be interpretable as lattice surfaces, (see e.g. 15, 7)). Regard the unit triangular lattice $\mathcal{T}$ on which the tiled region lives as being in a horizontal plane, and let $\mathcal{L}$ be a copy of the lattice $\left(\sqrt{\frac{3}{2}} \mathbb{Z}\right)^{3}$ placed so that one family of its body-diagonals is vertical, intersecting this plane at the vertices of $\mathcal{T}$. Then each segment joining two nearest neighbors of $\mathcal{L}$ projects onto a unit lattice segment of $\mathcal{T}$.

Orient the lattice segments of $\mathcal{T}$ so that they point in one of the polar directions $\pi / 2,-\pi / 6$, or $-5 \pi / 6$. Then we can lift a lozenge tiling of a lattice region on $\mathcal{T}$ by starting from some lattice point, tracing around its tiles one after another, and at each traversal of a lattice segment $s$ of $\mathcal{T}$, moving either up or down on the corresponding lattice segment of $\mathcal{L}$, according to whether the traversal respected or violated the orientation of $s$. Tracing around a lozenge results in going around a lattice square of $\mathcal{L}$ whose orthogonal projection on the plane of $\mathcal{T}$ is that lozenge.

If the tiling has no holes, the node of $\mathcal{L}$ we are finding ourselves at is independent of the way we traced around the tiles to get there. What results is a lattice surface in $\mathcal{L}$ whose lattice square faces are in one to one correspondence with the tiles.

When a hole of non-zero charge is present this ceases to be true. To illustrate this, suppose we have a left-pointing triangular hole of side two in our tiling (see Figure 5.1). Then as we trace its boundary counterclockwise, the traversal of each lattice segment agrees with its orientation. Thus, each complete turn results in six upward steps along lattice segments of $\mathcal{L}$, and leaves us at the node of $\mathcal{L}$ which is two cube-body-diagonals higher than where we started (this is illustrated, from three different viewpoints, in Figure 5.2). For a similar right-pointing hole we would end up two such diagonals lower.

In general, if we loop once counterclockwise along a closed walk through the lozenges of a tiling, the ending point of the corresponding walk on $\mathcal{L}$ is on the same vertical as its starting point, but a distance of $q \frac{3}{\sqrt{2}}$ below it, where $q$ is the total 


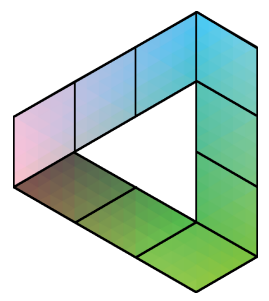

Figure 5.1. A tiling with a trianglular hole of size two.
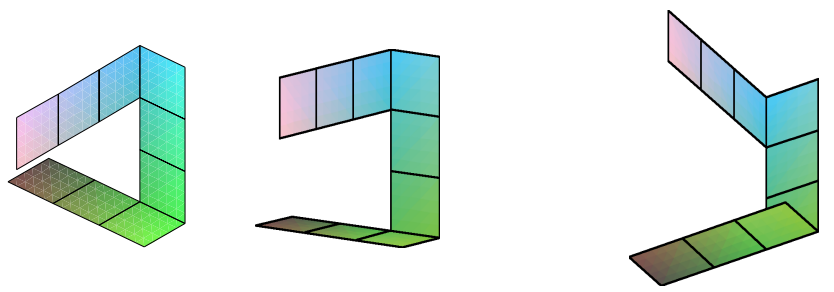

Figure 5.2. Three views of $\bar{S}_{t}$ for the tiling $T$ above.

charge of the holes we looped around (by a negative distance below we mean the absolute value of that distance above). This makes it impossible to get a singlesheeted lifting surface with no extra boundary in addition to the lifting of the boundary of the region with holes.

Nevertheless, we can construct a multi-sheeted surface with no additional boundary that lifts any tiling with holes, as follows. Suppose we have a lattice region $\mathcal{R}$ on $\mathcal{T}$ with a finite number of holes, and let $T$ be a lozenge tiling of it. For each hole, consider a fixed lattice path cut (independent of the tiling $T$ ) from it to the boundary of the region $\mathcal{R}$; let $\mathcal{P}$ be the family of lattice paths formed by them. Whenever a step of a lattice path of $\mathcal{P}$ crosses a lozenge of $T$, remove that lozenge from $\mathcal{R}$; let $\mathcal{R}_{0}$ be the region obtained from $\mathcal{R}$ by removing the union $T_{0}$ of all such lozenges of $T$. Now regard $T \backslash T_{0}$ as being a tiling of $\mathcal{R}_{0}$ in which $\mathcal{P} \cap \mathcal{R}_{0}$ is part of the boundary. Then $T \backslash T_{0}$ lifts to a single-sheeted lattice surface $6 \bar{S}_{T}$ in $\mathcal{L}$. The union 7

$$
U=\bigcup_{n \in \mathbb{Z}} \bar{S}_{T}+n \frac{3}{\sqrt{2}}
$$

is a multi-sheeted surface having holes above all the lozenges in $\mathcal{R} \backslash \mathcal{R}_{0}$. Define $S_{T}$ to be the multi-sheeted surface obtained from $U$ by filling in these holes with the missing square faces of the lattice $\mathcal{L}$ above the lozenges in $\mathcal{R} \backslash \mathcal{R}_{0}$. One readily sees that $S_{T}$ is independent of the family of cuts $\mathcal{P}$. The surface corresponding to the tiling in Figure 5.1 is illustrated in Figure 5.3. An instance with three holes is pictured in Figures 5.4 and 5.5.

\footnotetext{
${ }^{6}$ We assume here that the paths in $\mathcal{P}$ are disjoint. Unless the holes are packed very close (which, since we are interested in scaling limits, will not be the case for us), $\mathcal{P}$ can be chosen so.

${ }^{7}$ For a surface $S$ (single- or multi-sheeted) embedded in $\mathbb{R}^{3}$ and for $c \in \mathbb{R}$ we denote by $S+c$ the translation of $S$ by the vector $(0,0, c)$.
} 

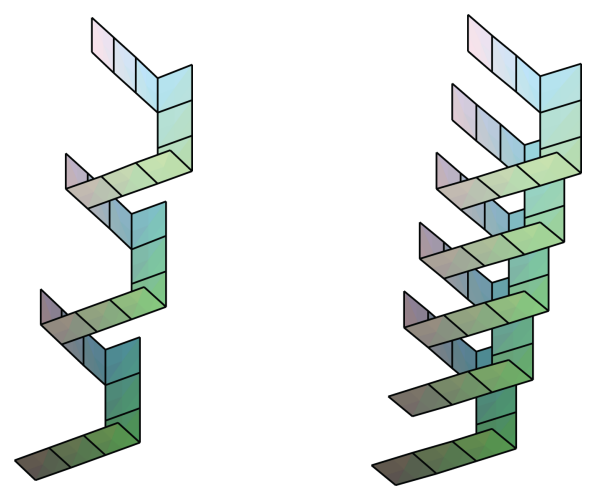

Figure 5.3. (a) One of the two connected components of $S_{T}$ for the tiling in Figure 5.1. (b) The full $S_{T}$.
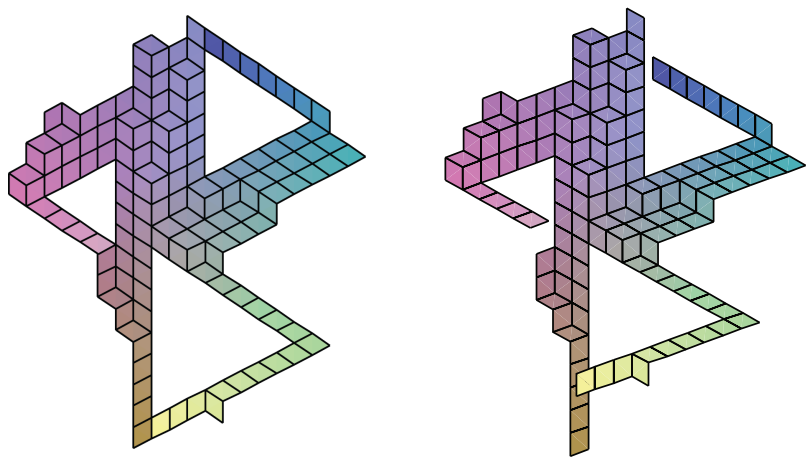

Figure 5.4. (a) A tiling $T$ with three holes. (b) A view of $\bar{S}_{T}$.

The detailed definition of the joint correlation $\hat{\omega}$ is the following (see $[3]$ ). For $j=1, \ldots, k$, let $Q_{j}$ be either a lozenge-hole or a lattice triangular hole of side two.

It is enough to define $\hat{\omega}\left(Q_{1}, \ldots, Q_{k}\right)$ when $q=\sum_{j=1}^{k} \operatorname{ch}\left(Q_{j}\right) \geq 0$ (the other case reduces to this by reflection across a vertical lattice line). Our definition is inductive on $q$ :

(i) If $q=0$, let $N$ be large enough so that the lattice rhombus of side $N$ centered at the origin encloses all $Q_{j}$ 's, and denote by $T_{N}$ the torus obtained from this large lattice rhombus by identifying its opposite sides. Set 8

$$
\hat{\omega}\left(Q_{1}, \ldots, Q_{k}\right):=\lim _{N \rightarrow \infty} \frac{\mathrm{M}\left(T_{N} \backslash Q_{1} \cup \cdots \cup Q_{k}\right)}{\mathrm{M}\left(T_{N}\right)} .
$$

(ii) If $q>0$, define

$$
\hat{\omega}\left(Q_{1}, \ldots, Q_{k}\right):=\lim _{R \rightarrow \infty} R^{q} \hat{\omega}\left(Q_{1}, \ldots, Q_{k}, W_{R, 0}\right) .
$$

The above limits exist by Proposition 2.1.

Let $\Delta_{1}, \ldots, \Delta_{k}$ be fixed lattice triangular holes of side two, and let $L$ be a fixed lozenge position. Assume $q=\sum_{j=1}^{k} \operatorname{ch}\left(\Delta_{j}\right)=0$. In the limit measure of the

${ }^{8} \mathrm{M}(\mathcal{R})$ denotes the number of lozenge tilings of the lattice region $\mathcal{R}$. 

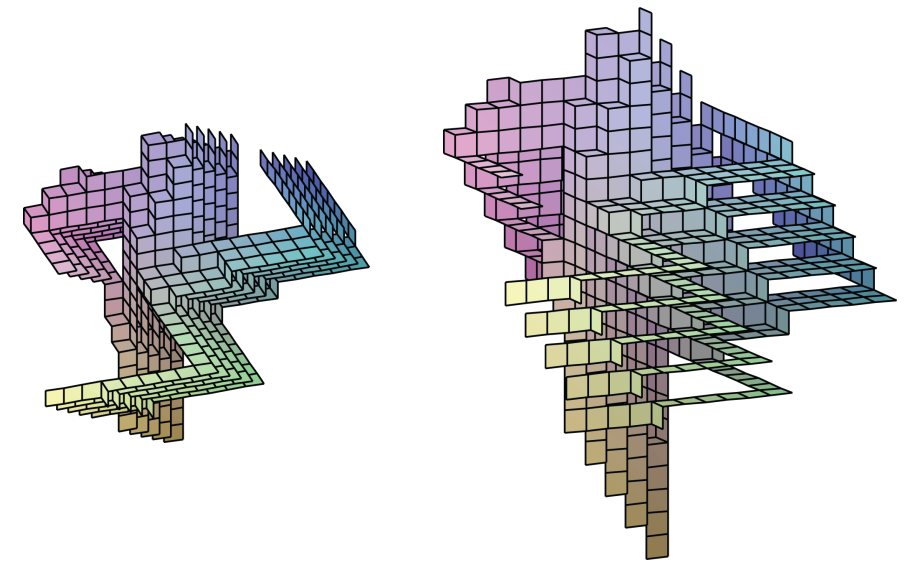

Figure 5.5. Two views of $S_{T}$ for the tiling $T$ in Figure 5.4(a).

uniform measures on the tori $T_{N} \backslash \Delta_{1} \cup \ldots \cup \Delta_{k}$, the probability that $L$ is occupied by a lozenge in a random tiling is

$$
\operatorname{Prob}\{L \text { is occupied }\}=\lim _{N \rightarrow \infty} \frac{\mathrm{M}\left(T_{N} \backslash L \cup \Delta_{1} \cup \cdots \cup \Delta_{k}\right)}{\mathrm{M}\left(T_{N} \backslash \Delta_{1} \cup \cdots \cup \Delta_{k}\right)}=\frac{\hat{\omega}\left(L, \Delta_{1}, \ldots, \Delta_{k}\right)}{\hat{\omega}\left(\Delta_{1}, \ldots, \Delta_{k}\right)} .
$$

This expression for the probability of $L$ being occupied holds in fact for general $q$. Indeed, suppose this has been established for total charges $<q$. Then the probability that $L$ is occupied in a random tiling with the extra hole $W_{R, 0}$ in addition to $\Delta_{1}, \ldots, \Delta_{k}$ is

$$
\frac{\hat{\omega}\left(L, \Delta_{1}, \ldots, \Delta_{k}, W_{R, 0}\right)}{\hat{\omega}\left(\Delta_{1}, \ldots, \Delta_{k}, W_{R, 0}\right)} .
$$

However, in the limit $R \rightarrow \infty$ this is, by (5.2), the same as the fraction on the right hand side of (5.3), and our statement is proved by induction.

Suppose $\mathcal{R}$ is a bounded simply connected lattice region on $\mathcal{T}$. Then each tiling of $\mathcal{R}$ lifts to a single-sheeted lattice surface on $\mathcal{L}$, and we can define the average lifting surface $S_{\text {av }}$ by taking the arithmetic mean of the finitely many heights of these surfaces above each node of $\mathcal{T}$. As pointed out in [7, if $u$ and $v$ are nearest neighbors in $\mathcal{T}$ so that the lattice segment between them is oriented from $u$ to $v$ (see the second paragraph of this section), then

$$
S_{\mathrm{av}}(v)-S_{\mathrm{av}}(u)=\frac{1}{\sqrt{2}}\left(1-3 p\left(L_{u v}\right)\right),
$$

where $L_{u v}$ is the lozenge location whose short diagonal is $u v$ (the factor multiplying the parenthesis on the right hand side arises because the traversal of each unit segment in $\mathcal{T}$ results in a change of height on the lifting surface of one third of a body diagonal of a lattice cube of $\mathcal{L}$ ). Thus, the height of $S_{\text {av }}$ at any node of $\mathcal{T}$ can be obtained by taking cumulative sums of lozenge occupation probabilities.

The regions we are concerned with - complements of finite unions of disjoint lattice triangular holes of side 2 - have an infinite set of lozenge tilings, so we cannot use the arithmetic mean as the definition of the average of the surfaces to which their tilings lift. However, since we know that the lozenge placement 
probabilities are given by (5.3), we can turn (5.4) around and use it to define this average surface.

More precisely, consider for each triangular hole a lattice path in $\mathcal{T}$ from a point on its boundary to infinity. Let $\mathcal{P}$ be the union of these lattice paths; assume they are disjoint. Define $\bar{S}_{\text {av }}$ to be the lattice surface on $\mathcal{L}$ satisfying

$$
\bar{S}_{\mathrm{av}}(v)-\bar{S}_{\mathrm{av}}(u)=\frac{1}{\sqrt{2}}\left(1-3 p\left(L_{u v}\right)\right),
$$

for any two nearest neighbors $u$ and $v$ of $\mathcal{T}$ for which the segment between them is oriented from $u$ to $v$ and is not crossed by any path of $\mathcal{P}$. Define the average lifting surface of the tilings of the complement of the holes by

$$
S_{\mathrm{av}}=\bigcup_{n \in \mathbb{Z}} \bar{S}_{\mathrm{av}}+n \frac{3}{\sqrt{2}} .
$$

This definition is readily seen to be independent of the family of cuts $\mathcal{P}$.

We note that in the case when there are no holes, the average surface can be defined in terms of the translation invariant ergodic measure on the hexagonal lattice (the lattice whose dimer coverings are equivalent to lozenge tilings of the triangular lattice), which follows by a general result of Sheffield (see [14, 13]) to be unique and given by a limit of uniform measures on tori. Due to the presence of holes our setting does not seem to fit that context.

The helicoid $H(a, b ; c)$ is the surface whose parametric equations in Cartesian coordinates are

$$
\begin{aligned}
& x=a+\rho \cos \theta, \\
& y=b+\rho \sin \theta, \quad-\infty<\rho, \theta<\infty . \\
& z=c \theta,
\end{aligned}
$$

The half helicoid $H^{+}(a, b ; c)$ is obtained by restricting the range of $\rho$ in the above to $(0, \infty)$. The dotted helicoid $\dot{H}(a, b ; c)$ is $H(a, b ; c)$ minus the vertical axis $x=$ $a, y=b$.

For positive integers $s$ define the $s$-refined half helicoid by

$$
H_{s}^{+}(a, b ; c):=\bigcup_{j=0}^{s-1} H^{+}(a, b ; c)+\frac{2 \pi c j}{s} .
$$

Define the $s$-refined dotted helicoid by

$$
\dot{H}_{s}(a, b ; c):=\bigcup_{j=0}^{s-1} \dot{H}(a, b ; c)+\frac{\pi c j}{s} .
$$

Note that for $s \in \mathbb{Z}$, the fibers of $H_{s}^{+}(a, b ; s c)$ above each point $u$ in the $x y$ coordinate plane are of the form $f(u)+2 \pi c \mathbb{Z}$. Thus, given $H_{s_{i}}^{+}\left(a_{i}, b_{i} ; s_{i} c\right), s_{i} \in \mathbb{Z}, i=1, \ldots, k$, one can define their sum

$$
S:=H_{s_{1}}^{+}\left(a_{1}, b_{1} ; s_{1} c\right)+\cdots+H_{s_{k}}^{+}\left(a_{k}, b_{k} ; s_{k} c\right)
$$

by defining the fiber of $S$ above $u$ to be

$$
f_{1}(u)+\cdots+f_{k}(u)+2 \pi c \mathbb{Z} .
$$

We can define the sum of dotted helicoids analogously, using the fact that the fibers of $\dot{H}_{s}(a, b ; s c)$ above $u$ are of the form $f(u)+\pi c \mathbb{Z}$. Figure 5.6(a) illustrates $H_{2}^{+}(0,0 ; 2)+H^{+}(1,0 ;-1)$. Note that a point on the surface making a complete 

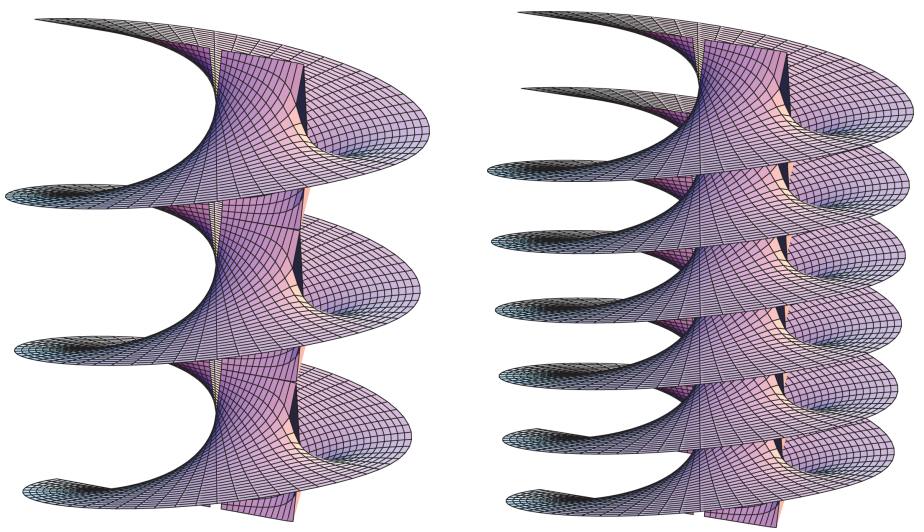

Figure 5.6. (a) $H_{2}^{+}(0,0 ; 2)+H^{+}(0,1 ;-1)$. (b) $H_{4}^{+}(0,0 ; 2)+$ $H_{2}^{+}(0,1 ;-1)$ is obtained by superimposing two copies of the surface (a).

counterclockwise turn around the "spiral stairwell" on the left ends up two levels higher; a similar turning around the other spiral takes the point one level lower. Figure 5.6(b) illustrates the effect of doubling the refinement indices in a sum of refined half helicoids: the new surface is the union of the original surface and a suitable vertical translate of it. A depiction of a sum of three helicoids is given in Figure 5.7.

Let $\mathcal{T}_{R}$ be the lattice obtained from the triangular lattice $\mathcal{T}$ by a homothety around the origin of factor $\frac{1}{R}$.

Theorem 5.1. Let $x_{1}^{(R)}, \ldots, x_{m}^{(R)}, y_{1}^{(R)}, \ldots, y_{m}^{(R)}, z_{1}^{(R)}, \ldots, z_{n}^{(R)}$ and $w_{1}^{(R)}, \ldots, w_{n}^{(R)}$ be sequences of integers so that $\lim _{R \rightarrow \infty} x_{i}^{(R)} / R=x_{i}, \lim _{R \rightarrow \infty} y_{i}^{(R)} / R=y_{i}$, $\lim _{R \rightarrow \infty} z_{j}^{(R)} / R=z_{j}$ and $\lim _{R \rightarrow \infty} w_{j}^{(R)} / R=w_{j}$ for $1 \leq i \leq m$ and $1 \leq j \leq n$. Assume the $\left(x_{i}, y_{i}\right)$ 's and $\left(z_{j}, w_{j}\right)$ 's are all distinct.

Let $E_{\mathbf{a}_{1}}^{q}, \ldots, E_{\mathbf{a}_{m}}^{q}$ and $W_{\mathbf{b}_{1}}^{q}, \ldots, W_{\mathbf{b}_{n}}^{q}, 3 \mid 1-q$, be multiholes on $\mathcal{T}_{R}$, and let the lists $\mathbf{a}_{i}$ and $\mathbf{b}_{j}$ have lengths $s_{i}$ and $t_{j}$, respectively. Let

$$
S_{\mathrm{av}}^{\mathcal{T}_{R}}=S_{\mathrm{av}}^{\mathcal{T}_{R}}\left(E_{\mathbf{a}_{1}}^{q}\left(x_{1}^{(R)}, y_{1}^{(R)}\right), \ldots, W_{\mathbf{b}_{n}}^{q}\left(z_{n}^{(R)}, w_{n}^{(R)}\right)\right)
$$

be the average lifting surface of the tilings of the complement of these multiholes on $\mathcal{T}_{R}$.

Then, as $R \rightarrow \infty, R S_{\mathrm{av}}^{\mathcal{T}_{R}}$ converges to the sum of refined helicoids

$$
\begin{aligned}
& \sum_{i=1}^{m} H_{2 s_{i}}^{+}\left(x_{i}, y_{i} ;-\frac{3 s_{i}}{\sqrt{2} \pi}\right)+\sum_{j=1}^{n} H_{2 t_{j}}^{+}\left(z_{j}, w_{j} ; \frac{3 t_{j}}{\sqrt{2} \pi}\right) \\
= & \sum_{i=1}^{m} \dot{H}_{s_{i}}\left(x_{i}, y_{i} ;-\frac{3 s_{i}}{\sqrt{2} \pi}\right)+\sum_{j=1}^{n} \dot{H}_{t_{j}}\left(z_{j}, w_{j} ; \frac{3 t_{j}}{\sqrt{2} \pi}\right) .
\end{aligned}
$$

For any bounded set $B$ and any open set $U$ containing $\left(x_{1}, y_{1}\right), \ldots,\left(x_{m}, y_{m}\right)$ and $\left(z_{1}, w_{1}\right), \ldots,\left(z_{n}, w_{n}\right)$, the convergence is uniform on $B \backslash U$.

Proof. In addition to our $60^{\circ}$ oblique coordinate system $\mathcal{O}$ in the plane of the unit triangular lattice $\mathcal{T}$, also consider a Cartesian system of coordinates $\mathcal{C}$ having the 

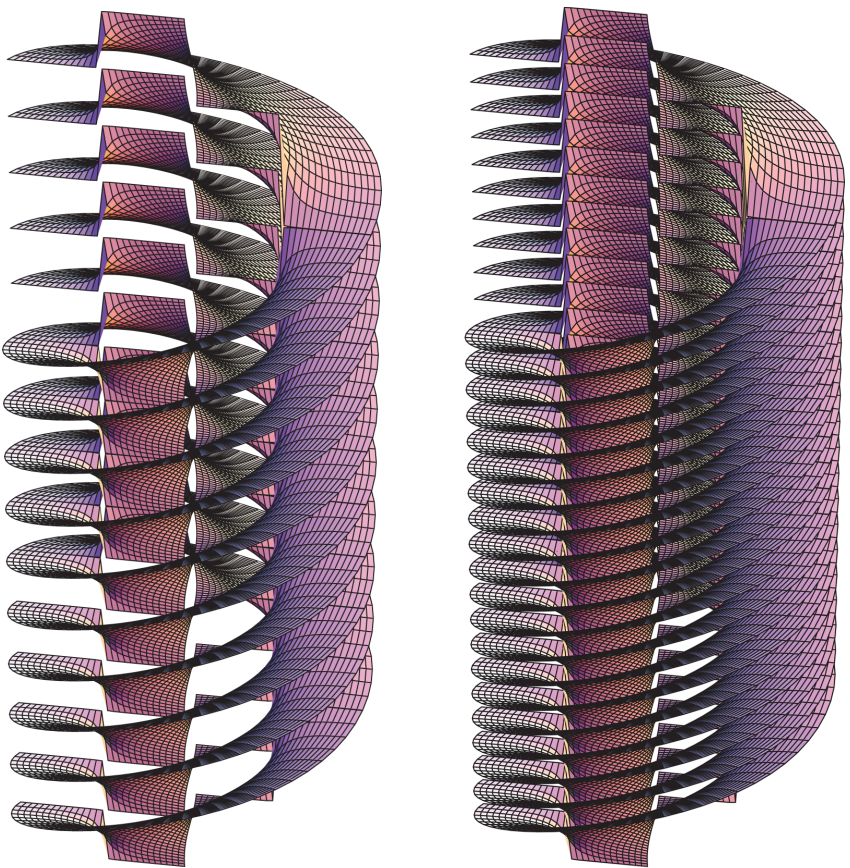

Figure 5.7. (a) The sum of the refined half helicoids $H_{2}^{+}(-1,0 ;-2), H_{3}^{+}(0,0 ; 3)$ and $H_{4}^{+}(1,0 ; 4)$. (b) Two copies of the latter give $H_{4}^{+}(-1,0 ;-2)+H_{6}^{+}(0,0 ; 3)+H_{8}^{+}(1,0 ; 4)$.

origin at the node of $\mathcal{T}$ just below the origin of $\mathcal{O}$, the $x$-axis in the polar direction 0 and the $y$-axis in the polar direction $\pi / 2$. One readily sees that the nodes of $\mathcal{T}$ have $\mathcal{C}$-coordinates $\left(a \frac{\sqrt{3}}{2}, b \frac{1}{2}\right), a, b \in \mathbb{Z}, a+b$ even, and that the midpoint of the segment connecting the nodes $\left(a \frac{\sqrt{3}}{2}, b \frac{1}{2}\right)$ and $\left(a \frac{\sqrt{3}}{2},(b+2) \frac{1}{2}\right)$ of $\mathcal{T}$ has $\mathcal{O}$ coordinates $\left(\frac{a-b}{2}, \frac{a+b}{2}\right)$.

We will use Cartesian coordinates to specify points at which $S_{\mathrm{av}}^{\mathcal{T}_{R}}$ is evaluated, and oblique coordinates for the lozenges whose placement probabilities come up.

We have by $(5.5)$

$$
\begin{aligned}
R \bar{S}_{\mathrm{av}}^{\mathcal{T}_{R}}\left(\frac{x_{0}^{(R)}}{R} \frac{\sqrt{3}}{2}, \frac{y_{0}^{(R)}+2}{R} \frac{1}{2}\right)-R & \bar{S}_{\mathrm{av}}^{\mathcal{T}_{R}}\left(\frac{x_{0}^{(R)}}{R} \frac{\sqrt{3}}{2}, \frac{y_{0}^{(R)}}{R} \frac{1}{2}\right) \\
= & \frac{1}{\sqrt{2}}\left(1-3 p_{1}\left(\frac{x_{0}^{(R)}-y_{0}^{(R)}}{2 R}, \frac{x_{0}^{(R)}+y_{0}^{(R)}}{2 R}\right)\right) \\
& =\frac{1}{\sqrt{2}}\left(1-3 p_{1}\left(\frac{x_{0}^{(R)}-y_{0}^{(R)}}{2}, \frac{x_{0}^{(R)}+y_{0}^{(R)}}{2}\right)\right),
\end{aligned}
$$

where $p_{1}$ is given by (2.12). The second equality holds because placement probabilities are clearly invariant under scaling. Analogous equations relate the change of 
$R \bar{S}_{\text {av }}^{\mathcal{T}_{R}}$ when moving a distance of $\frac{1}{R}$ from $\left(\frac{x_{0}^{(R)}}{R} \frac{\sqrt{3}}{2}, \frac{y_{0}^{(R)}}{R} \frac{1}{2}\right)$ in the polar directions $\frac{\pi}{2}+k \frac{\pi}{3}, k \in \mathbb{Z}$.

The asymptotics of $p_{1}\left(x_{0}^{(R)}, y_{0}^{(R)}\right)-p_{2}\left(x_{0}^{(R)}, y_{0}^{(R)}\right)$ follows by (3.1) and (1.2). One obtains the asymptotics of $p_{1}\left(x_{0}^{(R)}, y_{0}^{(R)}\right)-p_{3}\left(x_{0}^{(R)}, y_{0}^{(R)}\right)$ similarly from (1.3). Adding up the two and using $p_{1}+p_{2}+p_{3}=1$ yields

$$
\begin{aligned}
& 1-3 p_{1}\left(x_{0}^{(R)}, y_{0}^{(R)}\right)=-\frac{3 \sqrt{3}}{2 \pi}\left\{\sum_{i=1}^{m} s_{i} \frac{x_{0}-x_{i}+y_{0}-y_{i}}{\left(x_{0}-x_{i}\right)^{2}+\left(x_{0}-x_{i}\right)\left(y_{0}-y_{i}\right)+\left(y_{0}-y_{i}\right)^{2}}\right. \\
& \begin{aligned}
(5.12) \\
\left.\quad-\sum_{j=1}^{n} t_{j} \frac{x_{0}-z_{j}+y_{0}-w_{j}}{\left(x_{0}-z_{j}\right)^{2}+\left(x_{0}-z_{j}\right)\left(y_{0}-w_{j}\right)+\left(y_{0}-w_{j}\right)^{2}}\right\} \frac{1}{R}+o\left(\frac{1}{R}\right),
\end{aligned}
\end{aligned}
$$

with the implicit constant uniform for $\left(x_{0}, y_{0}\right) \in B \backslash U$. Similar expressions follow for the asymptotics of $p_{2}$ and $p_{3}$.

This allows us to deduce that $\lim _{R \rightarrow \infty} R \bar{S}_{\mathrm{av}}^{\mathcal{T}_{R}}$ exists. Indeed, the height of $R \bar{S}_{\mathrm{av}}^{\mathcal{T}_{R}}$ at any node of $\mathcal{T}_{R}$ can be obtained by taking cumulative sums of (5.11) and its two analogs. By (5.12) and its analogs, the resulting error terms have a zero effect in the limit $R \rightarrow \infty$ : even though they do add up, and there are more and more of them as $R$ gets large, since the individual errors are small and the factor $\frac{1}{R}$ is present, the combined contribution of the error terms is small.

Furthermore, we can find the gradient of the limiting surface $\mathcal{S}$ by dividing the left hand side of (5.11) by $\frac{1}{R}$ - the distance between the two points where the heights of $R \bar{S}_{\mathrm{av}}^{\mathcal{T}_{R}}$ are examined — and letting $R \rightarrow \infty$. This yields

$$
\begin{aligned}
\frac{\partial \mathcal{S}}{\partial y}\left(x_{0} \frac{\sqrt{3}}{2}, y_{0} \frac{1}{2}\right)=-\frac{3}{\sqrt{2} \pi}\left\{\sum_{i=1}^{m} s_{i} \frac{\frac{\sqrt{3}}{2}\left(x_{0}-x_{i}\right)}{\frac{3}{4}\left(x_{0}-x_{i}\right)^{2}+\frac{1}{4}\left(y_{0}-y_{i}\right)^{2}}\right. \\
\left.-\sum_{j=1}^{n} t_{j} \frac{\frac{\sqrt{3}}{2}\left(x_{0}-z_{j}\right)}{\frac{3}{4}\left(x_{0}-z_{j}\right)^{2}+\frac{1}{4}\left(y_{0}-w_{j}\right)^{2}}\right\}
\end{aligned}
$$

for $\left(x_{0}, y_{0}\right)$ different from $\left(x_{1}, y_{1}\right), \ldots,\left(x_{m}, y_{m}\right)$ and $\left(z_{1}, w_{1}\right), \ldots,\left(z_{n}, w_{n}\right)$. After a change of variables this becomes

$$
\begin{aligned}
\frac{\partial \mathcal{S}}{\partial y}\left(x_{0}, y_{0}\right)=-\frac{3}{\sqrt{2} \pi}\left\{\sum_{i=1}^{m} s_{i} \frac{\left(x_{0}-x_{i}\right)}{\left(x_{0}-x_{i}\right)^{2}+\left(y_{0}-y_{i}\right)^{2}}\right. \\
\left.-\sum_{j=1}^{n} t_{j} \frac{\left(x_{0}-z_{j}\right)}{\left(x_{0}-z_{j}\right)^{2}+\left(y_{0}-w_{j}\right)^{2}}\right\} .
\end{aligned}
$$

The analog of (5.11) corresponding to moving one unit in the polar direction $\frac{\pi}{6}$ yields by a similar calculation the directional derivative of $\mathcal{S}$ along this polar direction. Writing $\partial / \partial x$ as the appropriate linear combination of this directional derivative 
and $\partial / \partial y$ we arrive at

$$
\begin{aligned}
\frac{\partial \mathcal{S}}{\partial x}\left(x_{0}, y_{0}\right)=\frac{3}{\sqrt{2} \pi}\left\{\sum_{i=1}^{m}\right. & s_{i} \frac{\left(y_{0}-y_{i}\right)}{\left(x_{0}-x_{i}\right)^{2}+\left(y_{0}-y_{i}\right)^{2}} \\
& \left.-\sum_{j=1}^{n} t_{j} \frac{\left(y_{0}-w_{j}\right)}{\left(x_{0}-z_{j}\right)^{2}+\left(y_{0}-w_{j}\right)^{2}}\right\} .
\end{aligned}
$$

On the other hand, the gradient at $(x, y)$ of the half helicoid $H^{+}(a, b ; c)$ - and hence of any refinement of it - is $\left(-\frac{c(y-b)}{(x-a)^{2}+(y-b)^{2}}, \frac{c(x-a)}{(x-a)^{2}+(y-b)^{2}}\right)$. Thus, if $\mathcal{H}$ is the sum of refined helicoids

$$
\mathcal{H}=\sum_{i=1}^{m} H_{2 s_{i}}^{+}\left(x_{i}, y_{i} ;-\frac{3 s_{i}}{\sqrt{2} \pi}\right)+\sum_{j=1}^{n} H_{2 t_{j}}^{+}\left(z_{j}, w_{j} ; \frac{3 t_{j}}{\sqrt{2} \pi}\right),
$$

we have that the gradients of $\mathcal{H}$ and $\mathcal{S}$ agree on $\mathbb{R}^{2} \backslash\left\{\left(x_{1}, y_{1}\right), \ldots,\left(z_{n}, w_{n}\right)\right\}$.

By construction, each lifting surface of a tiling of the complement of our multiholes has fibers of the form $c+\frac{3}{\sqrt{2}} \mathbb{Z}$. Thus so does $R \bar{S}_{\mathrm{av}}^{\mathcal{T}_{R}}$ for all $R$, and also the limit surface $\mathcal{S}$. The same is true for each summand in (5.15), and therefore for $\mathcal{H}$. Then the surface $\mathcal{S}_{0}=\mathcal{S}-\mathcal{H}$ is well defined by (5.9). By construction, as $u$ approaches $\left(x_{1}, y_{1}\right)$ the fiber of $\mathcal{S}$ above $u$ approaches $0+\frac{3}{\sqrt{2}} \mathbb{Z}$. It follows from the definition of $\mathcal{H}$ that the same is true for the fibers of $\mathcal{H}$. This, together with the fact that its gradient is zero, implies that $\mathcal{S}_{0}$ is the surface $0+\frac{3}{\sqrt{2}} \mathbb{Z}$. So $\mathcal{S}=\mathcal{H}$, justifying the first expression in (5.10). The second expression follows using the elementary fact that $H^{+}(a, b ; c) \cup\left(H^{+}(a, b ; c)+\frac{c}{2}\right)=\dot{H}(a, b ; c)$ (an instance of this can be seen in Figure 5.6(a)). The stated uniformity of the convergence is implied by the uniformity of the error terms in (5.12) and its analogs.

\section{Physical interpretation}

The results of 2 and 3 already show a close connection between random lozenge tilings with holes and electrostatics: the joint correlation of holes is given, in the scaling limit, by the electrostatic energy of the corresponding system of electrical charges. Theorem 1.1 strengthens this connection by showing that the electrostatic field itself can be viewed as the scaling limit of the discrete field of the average orientation of lozenges in a random tiling of the complement of the holes.

Following [2, §2], suppose one pictures the two dimensional universe as being spatially quantized, consisting of a very fine lattice of a triangular quanta of space.

Associate the complement of the holes with the vacuum of empty space. The quantum fluctuations of the vacuum cause virtual electron-virtual positron pairs to be ceaselessly created and annihilated. Associate virtual electrons with left-pointing unit triangles and virtual positrons with right-pointing unit triangles. Then a lozenge corresponds to the annihilation of a virtual electron-virtual positron pair, and a lozenge tiling to one way for all virtual electron-virtual positron pairs to annihilate.

Averaging over all possible tilings then arises naturally as corresponding to the Feynman sum over all possible ways the virtual electrons and virtual positrons can annihilate in pairs. Our result states that if the distances between the hole-charges and the distances between them and the point where we are measuring the $\mathbf{F}$-field 
are much larger than the lattice spacing of the quantas of space, then this field is almost exactly the same as the electric field. From this perspective, one sees as the result of an exact calculation how the (two dimensional) electric field emerges from the quantum fluctuations of the vacuum. This view provides an electric field which very closely approximates the Coulomb field, but is not exactly equal to it: by Theorem 1.1, the latter occurs only in the limit when the size of the quantas of space approaches zero. The discrepancy gets noticeable only at very small distances. This is in agreement with the expectation of some physicists that linear superposition for the electric field might break down in the subatomic domain (see, e.g. Jackson [11, §I.3]).

In quantum mechanics, when a virtual electron and a virtual positron annihilate, two photons result, which are sent away in opposite directions. The unit contribution to $\mathbf{F}(e)$ of each lozenge covering $e$ in a tiling could be regarded as corresponding to one of these two photons (this assumes that the common direction of the two ejected photons is, on average, the straight line connecting the annihilating virtual particles). Then Theorem 1.1 states that in the scaling limit, the thus generated "jet" of photons at $e$ averages precisely to the electrostatic field. This could then be viewed as a "mechanism" for the coming about of the electrostatic field.

The discussion in the previous paragraph leaves the second photon unaccounted for. However, underlying in our considerations is a second discrete vector field we have not yet mentioned: the one given by the average orientation of the lozenge that covers any fixed right-pointing unit triangle! Denote it by $\mathbf{F}^{\prime}$. An argument similar to the one that proved Theorem 1.1 shows that $\mathbf{F}^{\prime}=-\mathbf{F}$ in the scaling limit.

We conclude by mentioning another, quite different way to define a vector field via random tilings with holes. Let $Q_{1}, \ldots, Q_{n}$ be a fixed collection of holes on the unit triangular lattice. For any $x, y, \alpha, \beta \in \mathbb{Z}$ define

$$
T_{\alpha, \beta}(x, y):=\frac{1}{\sqrt{\alpha^{2}+\alpha \beta+\beta^{2}}}\left(\frac{\hat{\omega}\left(E_{x+\alpha, y+\beta}, Q_{1}, \ldots, Q_{n}\right)}{\hat{\omega}\left(E_{x, y}, Q_{1}, \ldots, Q_{n}\right)}-1\right)
$$

(i.e., the hole $E_{x, y}$ plays the role of a "test charge," and the effects of its displacement are recorded). Then it can be shown (details will appear in a separate paper) that there exists a vector field $\mathbf{T}$ so that in the scaling limit $T_{\alpha, \beta}(x, y)$ is the orthogonal projection of $\mathbf{T}(x, y)$ onto the vector $(\alpha, \beta)$. Furthermore, up to a constant multiple, the field $\mathbf{T}$ turns out to be the same as $\mathbf{F}$. Thus, unlike in physics where the electric field is defined by means of a test charge, in our model there are two different ways to define the corresponding field, and one of them does not use test charges.

We note that the agreement in the previous paragraph is not a matter of course, as it does not hold for instance on the critical Fisher lattice - the planar lattice of equilateral triangles and regular dodecagons where the edges of the triangles have weight 1 and the inter-triangle edges have weight $\sqrt{3}$ (this follows from not yet published joint work with David Wilson).

\section{REFERENCES}

1. M. Ciucu, Rotational invariance of quadromer correlations on the hexagonal lattice, Adv. in Math. 191 (2005), 46-77. MR2102843 (2006j:52023)

2. M. Ciucu, A random tiling model for two dimensional electrostatics, Mem. Amer. Math. Soc. 178 (2005), no. 839. MR2172582(2007c:82022) 
3. M. Ciucu, The scaling limit of the correlation of holes on the triangular lattice with periodic boundary conditions, Mem. Amer. Math. Soc. 199 (2009), no. 935, 1-100. arXiv:math$\mathrm{ph} / 0501071$.

4. M. Ciucu, Dimer packings with gaps and electrostatics, Proc. Natl. Acad. Sci. USA 105 (2008), 2766-2772. MR2383565 (2009a:82012)

5. H. Cohn, N. Elkies, and J. Propp, Local statistics for random domino tilings of the Aztec diamond, Duke Math. J. 85 (1996), 117-166. MR.1412441 (97k:52026)

6. H. Cohn, R. Kenyon, and J. Propp, A variational principle for domino tilings, J. Amer. Math. Soc. 14 (2001), 297-346. MR.1815214 (2002k:82038)

7. H. Cohn, M. Larsen, and J. Propp, The shape of a typical boxed plane partition, New York J. of Math. 4 (1998), 137-165. MR1641839 (99j:60011)

8. T. H. Colding and W. P. Minicozzi II, Disks that are double spiral staircases, Notices Amer. Math. Soc. 50 (2003), 327-339. MR1954009 (2004i:53010)

9. R. P. Feynman, "QED: The strange theory of light and matter," Princeton University Press, Princeton, New Jersey, 1985.

10. M. E. Fisher and J. Stephenson, Statistical mechanics of dimers on a plane lattice. II. Dimer correlations and monomers, Phys. Rev. (2) 132 (1963), 1411-1431. MR0158705 (28:1928)

11. J. D. Jackson, "Classical Electrodynamics," Third Edition, Wiley, New York, 1998. MR0436782 (55:9721)

12. R. Kenyon, Local statistics of lattice dimers, Ann. Inst. H. Poincaré Probab. Statist. 33 (1997), 591-618. MR 1473567 (99b:82039)

13. R. Kenyon, A. Okounkov, and S. Sheffield, Dimers and Amoebae, Ann. of Math. 163 (2006), 1019-1056. MR2215138 (2007f:60014)

14. S. Sheffield, Random Surfaces, Astérisque, 2005, no. 304. MR2251117 (2007g:82021)

15. W. P. Thurston, Conway's tiling groups, Amer. Math. Monthly 97 (1990), 757-773. MR.1072815 (91k:52028)

Department of Mathematics, Indiana University, Bloomington, Indiana 47405 